\title{
TOTAL LEAST SQUARES AND CONSTRAINED \\ LEAST SQUARES APPLIED TO FREQUENCY \\ DOMAIN SYSTEM IDENTIFICATION
}

\begin{abstract}
A Thesis Presented to
The Faculty of the College of Engineering and Technology

Ohio University
\end{abstract}

\begin{abstract}
In Partial Fulfillment
of the Requirements for the Degree

Master of Science
\end{abstract}

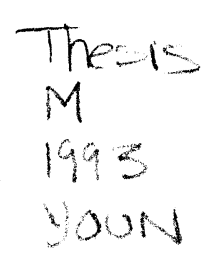

by

William Ronald Young,

March, 1993

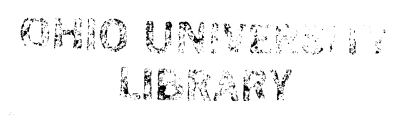


Acknowledgements

The author wishes to take this opportunity to thank the many people without whose help this thesis would not have been possible.

Thanks go to Dr. R. Dennis Irwin for his support, technical advice, guidance, and supervision. Also, the author is grateful to Dr. Irwin and Dr. Jerrel Mitchell for their helpful input and insight learned through years of experience in the field.

Thanks also go to the other Professors in the Electrical and Computer Engineering Department of the College of Engineering and Technology for the advanced education and experience that has been received as a part of the author's graduate program. Their contributions to the author's education are greatly appreciated. Most importantly, the author wishes to thank Paul and Beth Stocker whose endowment to Ohio University helped financed this graduate program. Were it not for this funding, this degree would never have been possible. 
Table of Contents

Acknowledgements $\ldots \ldots \ldots \ldots \ldots \ldots \ldots \ldots \ldots \ldots \ldots \ldots$

Table of Contents $\ldots \ldots \ldots \ldots \ldots \ldots \ldots \ldots \ldots$ ii

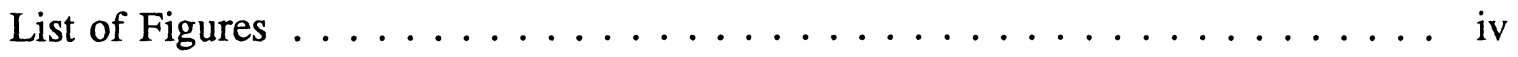

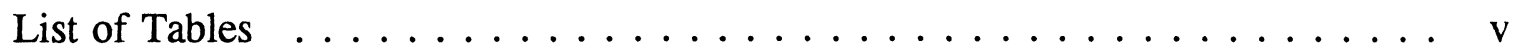

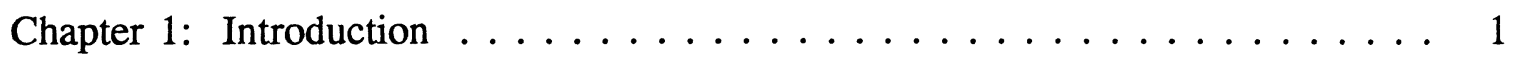

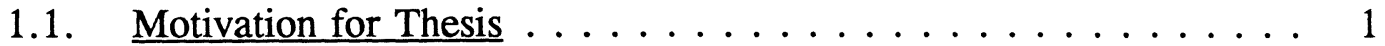

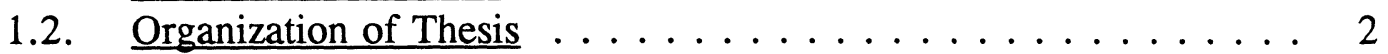

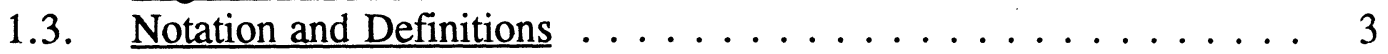

Chapter 2: TLS and CLS Solutions to Linear Equations . . . . . . . . . . 6

2.1. Total Least Squares (TLS) . . . . . . . . . . . . . . . 7

2.1.1. The Basic TLS Problem ................ . . . . . . . . .

2.1.2. The mixed LS/TLS problem .............. . . . . . . . . . . . 11

2.2. Constrained Least Squares (CLS) . . . . . . . . . . . . . . . 14

Chapter 3: Transfer Function Determination Code (TFDC) . . . . . . . . . . 17

3.1. Derivation of TFDC Equations ................. 18

3.2. Ordinary Least Squares (LS) Solution and the TFDC Algorithm . . 20

3.3. The TFDC Code (Usage and Modifications) . . . . . . . . . . 22

3.3.1. Speed Improvement . . . . . . . . . . . . . . 23

3.3.2. Constraint Equations for the CLS Solution Method . . . . 26

3.3.3. Algorithms Used in TLS, LS/TLS, and CLS solutions . . . 29

Chapter 4: System Identification Using Modified TFDC . . . . . . . . . . . . 32

4.1. Description of Data Used to Test the Modified TFDC Code . . . 32

4.2. Using Matlab's SIMULAB Toolbox for the Linear Simulation ... 35

4.3. Obtaining Frequency Response Data From Time Sequences ... . 38

4.4. Results from Applying TFDC to the Constructed Example System . . . . . . . . . . . . . . . . . . . . 39

4.5. Results from Applying TFDC to the AGS Subsystem Data .... 44

Chapter 5: Conclusions and Recommendations . . . . . . . . . . . . 48

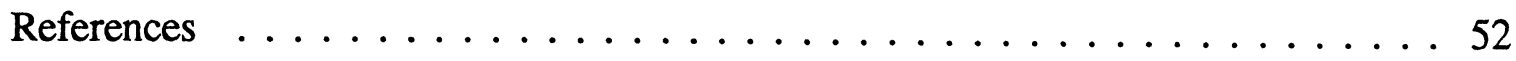

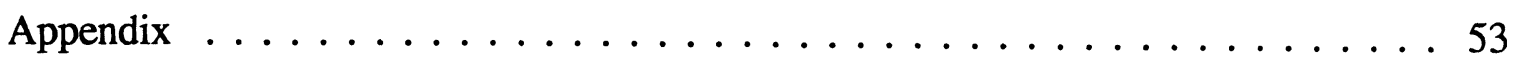




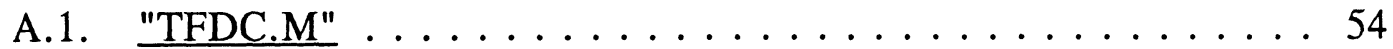

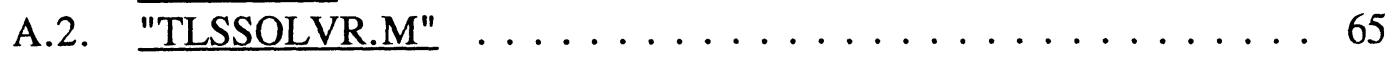

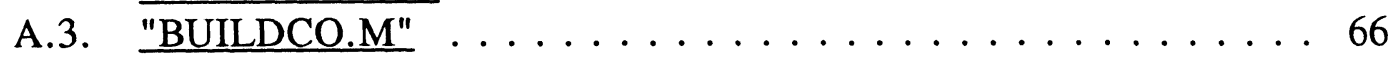

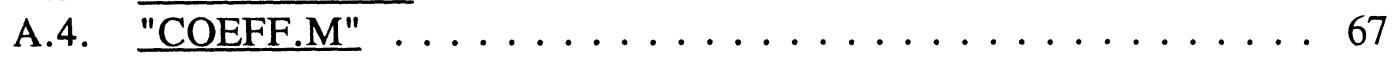

A.5. "BINOM.M" ................... 68 
List of Figures

Figure 4.1: Analytical Frequency Response for the Constructed System,

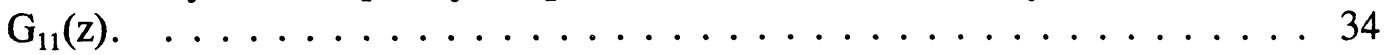

Figure 4.2: Analytical Frequency Response for the Constructed System, $\mathrm{G}_{12}(\mathrm{z})$.

Figure 4.3: Analytical Frequency Response for the Constructed System,

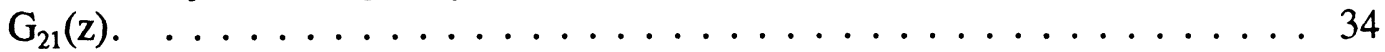

Figure 4.4: Analytical Frequency Response for the Constructed System,

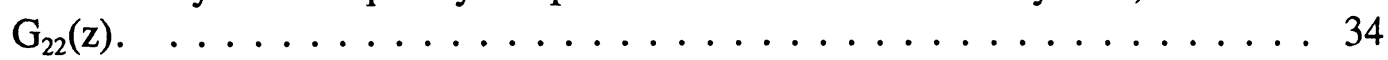

Figure 4.5: Marshall Space Flight Center Large Space Structure Ground Test

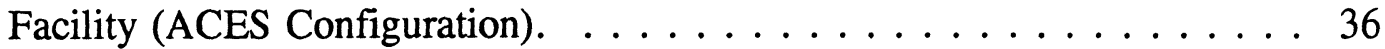

Figure 4.6: Block diagram of simulation using Matlab SIMULAB toolbox. . . 37

Figure 4.7: Complex Transfer Function System I.D. (LS Solution). . . . . . . . 39

Figure 4.8: Complex Transfer Function System I.D. (TLS Solution). . . . . . . . 40

Figure 4.9: Complex Transfer Function System I.D. (LS/TLS Solution). . . . . . 40

Figure 4.10: Power Spectral Density System I.D. (LS Solution). . . . . . . . . . . 43

Figure 4.11: Power Spectral Density System I.D. (TLS Solution). . . . . . . . . 43

Figure 4.12: Power Spectral Density System I.D. (CLS Solution). . . . . . . . . . 44

Figure 4.13: Complex Transfer Function System I.D. AGSx to BGx. (LS

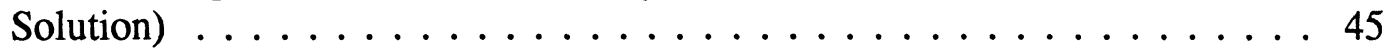

Figure 4.14: Complex Transfer Function System I.D. AGSx to BGx (TLS Solution). . . . . . . . . . . . . . . . . . . 46

Figure 4.15: Complex Transfer Function System I.D. AGSy to BGy (LS

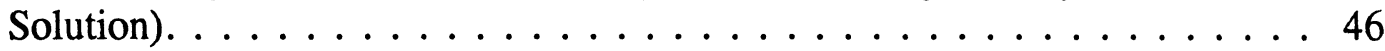

Figure 4.16: Complex Transfer Function System I.D. AGSy to BGy (TLS Solution). . . . . . . . . . . . . . . . . . 47 
List of Tables

Table 3.1: Comparison of Frequency Selection Times . . . . . . . . . . . 24

Table 3.2: Comparison of Times to Build Linear Equations . . . . . . . . . . 27

Table 4.1: Comparison of coefficients determined from TLS and LS/TLS

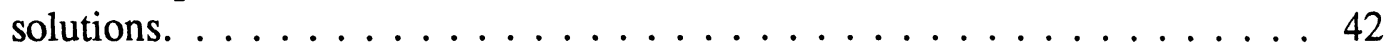




\section{Chapter 1: Introduction}

Many of the classical and modern controller design techniques require an accurate linear model of the plant . The Transfer Function Determination Code (TFDC) is a system identification algorithm for determining the input-output transfer function for single-input, single-output (SISO) discrete-time systems. TFDC determines a SISO system transfer function using input data obtained from experimental frequency response calculations of the discrete-time system. Given that the frequency response data contains $N$ frequency points, the code manipulates the frequency response input data into a set of $2 N$ linear equations in $2 n+1$ unknowns where $n$ is the assumed order of the resulting input-output transfer function. In practice, generally $N \gg n$, thus TFDC generates a greatly overdetermined set of linear equations. The current version of TFDC finds an ordinary Least Squares (LS) solution to these equations with the solution vector containing the coefficients of the assumed transfer function model.

\subsection{Motivation for Thesis}

As mentioned above, many controller design techniques in use today require an accurate and reliable linear model of the plant to be controlled. This thesis arises from a need to improve the TFDC code so that more accurate and dependable models will be realized with less effort by the user. Currently, TFDC solves the system of equations using an iterative, weighted ordinary LS solution. The LS solution without weighting or iteration rarely produces an accurate model even when given frequency 
data that is relatively noise free. To improve the model accuracy, a weighted least squares solution was implemented and the identification process was made iterative. This improved the resulting models but also added much more user interaction and decision making in the code execution. First, the user must decide the method(s) of coefficient matrix weighting to use. Several weighting methods have been examined, but no consistent method has been found which will work for a number of systems. One method works well for one system and another method works well for a different set of system data. Second, the user must decide when to stop the iteration process built into the code. Thus the current TFDC code requires a significant amount of trial and error in finding the best combination of weighting and number of iterations to find an accurate system model. In addition, TFDC has not produced an acceptable model for some experimental data even with weighting and iteration. The purpose of this work is to implement Total Least Squares (TLS), combined Least Squares/Total Least Squares (LS/TLS), and Constrained Least Squares (CLS) solutions to the system of equations developed in TFDC, to apply TFDC using these solution methods to system data, and to analyze the results of using these solution methods.

\subsection{Organization of Thesis}

This thesis contains five chapters and one appendix. Chapter 1 is this introduction which gives an overview of this thesis and explains the motivation for this work. Section 1.3 describes the notation, assumptions, and definitions used. The theory and mathematical background for the TLS and CLS solution methods is presented in chapter 2. Included in the presentation of TLS is a variation which 
combines a LS and TLS solution which is, theoretically, the best suited least squares method for the TFDC algorithm. Chapter 3 describes the TFDC algorithm. First, the system of linear equations are derived. Second, Section 3.2 contains is a summary of the results and problems from TFDC using the LS solution. Finally, Section 3.3 includes a discussion of the TFDC code modifications which include speed improvements, addition of user selection of the solution method used, and algorithms for implementing the TLS, LS/TLS, and CLS solution methods. Chapter 4 is devoted to applying the improved TFDC code to a constructed example system and to experimental data obtained from the NASA Marshall Space Flight Center in Huntsville, Alabama. Chapter 4 begins with a description of the constructed system and an explanation of the data from NASA Marshall. The methods used in simulating the constructed system and obtaining frequency response data from time records are described in Sections 4.2 and 4.3. Section 4.4 presents the results of using TFDC on data from the constructed system. Finally, the results of applying TFDC to the NASA data is presented. Chapter 5 discusses conclusions and recommendations for future work.

\subsection{Notation and Definitions}

This section introduces the notation, assumptions, and definitions used throughout this work.

- Matrices are denoted by capital letters, e.g. A. Columns and elements of a matrix are denoted by the corresponding lowercase letter with single or 
double subscripts, e.g. $a_{i}$ denotes the $i$ th column of $A$ and $a_{i j}$ denotes the $(i, j)$ th element of $A$.

- Vectors are denoted by lowercase letters, e.g. b. Components of a vector are denoted with single subscripts, e.g. $b_{i}$ is the $i$ th component of the vector $b$.

- Submatrices will denoted with the colon notation used by Golub and Van Loan (1989) and The Mathworks, Inc. (1990).

- The transpose of a vector or matrix is denoted by a superscript T, e.g. $A^{T}$.

- $\mathrm{R}(A)$ and $\mathrm{N}(A)$ denote the range space and null space, respectively of the matrix $A$.

- Diagonal matrices will be specified with the following notation.

$$
A=\operatorname{diag}\left(\alpha_{1}, \cdots, \alpha_{p}\right), \quad p=\min \{m, n\}
$$

denotes that $A$ is an $m \times n$ matrix where $a_{i j}=0$ when $i \neq j$ and $a_{i i}=$ $\alpha_{i}$ for $i=1, \cdots, p$.

- An $m \times m$ identity matrix is denoted by $I_{m}$.

- An $m \times n$ zero matrix is denoted by $0_{m \times n}$.

- The Frobenius norm of an $m \times n$ matrix $A$ is defined by

$$
\|A\|_{F}=\sqrt{\sum_{i=1}^{m} \sum_{j=1}^{n} a_{i j}^{2}} .
$$

- The 2-norm of a $n$-dimensional vector $x$ is defined by

$$
\|y\|_{2}=\sqrt{\sum_{i=1}^{n} y_{i}^{2}} .
$$


- The rank of the matrix $A$ is denoted by $\operatorname{rank}(A)$ and defined by the number of its non-zero singular values.

- Matlab commands will be enclosed in double quotation marks, e.g. "spectrum". References to variable names in the Matlab workspace or command line will be in boldface type. 


\section{Chapter 2: TLS and CLS Solutions to Linear Equations}

System identification in general, and specifically the Transfer Function Determination Code (TFDC), often involves the solution of a system of linear equations, $A x \approx b$, which is inconsistent. This system of equations is almost always an overdetermined set of linear equations. A variety of least squares methods can be used to find a numerical solution to these equations. Which methods will yield the best results depends heavily on the error present in the data and the statistical properties of the error. TFDC is no exception. In the case where the errors are confined to $b$, ordinary Least Squares (LS) is the solution method generally used; i.e., finding $\hat{x}$ such that $\|A \hat{x}-\hat{b}\|_{2}$ is minimum. However, when $A$ and $b$ are both subject to error or uncertainty, as is the case with the system of equations that arises in TFDC, the Total Least Squares (TLS) solution can give a better solution than ordinary LS solutions. Presently, TFDC implements an ordinary LS solution to the linear system. This thesis investigates the application of the Total Least Squares (TLS) and Constrained Least Squares (CLS) methods to the TFDC problem. Before deriving the TFDC equations in Chapter 3, this chapter will present the necessary background material on TLS and CLS. This chapter first introduces the basic TLS problem and solution in Section 2.1.1. Section 2.1.2 presents the mixed LS/TLS problem, a variation on the ordinary TLS problem. Finally, the CLS problem and solution are given in Section 2.2. 


\subsection{Total Least Squares (TLS)}

The basic principle of TLS is that the noisy data $[A ; b]$, while not satisfying a linear relation, i.e. $A x=b$, are modified with minimal effort, as measured by the Frobenius norm and 2-norm, to a "nearby" matrix $[\hat{A} ; \hat{b}]$ that is rank-deficient so that the set $\hat{A} x=\hat{b}$ is consistent. (Van Huffel and Vandewalle, 1991, p. 46) Besides the basic TLS problem, there also exist several variations and generalizations of the basic TLS problem. This discussion will only consider the one-dimensional (i.e. only one right-hand side vector) problem since this is the case that applies to TFDC. Also, as only the basic TLS problem and the mixed LS/TLS problem are applied to TFDC, none of the other TLS variations or generalizations will be discussed.

\subsubsection{The Basic TLS Problem}

The basic TLS problem can be formally defined as follows:

Definition 2.1. (basic TLS problem) Given an overdetermined set of $m$ linear equations $A x \approx b$ in $n$ unknowns $x(\mathrm{~m}>\mathrm{n})$, the total least squares problem seeks to

$$
\begin{gathered}
\underset{[\hat{A} ; \hat{b}] \in \mathbf{R}^{\times \times(x+1)}}{\operatorname{minimize}}\|[A ; b]-[\hat{A} ; \hat{b}]\|_{F} \\
\text { subject to } \hat{b} \in R(\hat{A}) .
\end{gathered}
$$

Once a minimizing $[\hat{A} ; \hat{b}]$ is found, then any $\hat{x}$ satisfying

$$
\hat{A} \hat{x}=\hat{b}
$$

is defined as a TLS solution and $[\Delta \hat{A} ; \Delta \hat{b}]=[A ; b]-[\hat{A} ; \hat{b}]$ as the corresponding TLS correction.

(Van Huffel and Vandewalle, 1991, pp.33-34) 
Two theorems will be needed in deriving the solution to the basic TLS

problem.

Theorem 2.1. (Eckart-Young-Mirsky matrix approximation theorem) Let the SVD of $C \in \mathbb{R}^{m \times n}$ be given by $C=\sum_{i=1}^{r} \sigma_{i} u_{i} v_{i}^{T}$ where $r=\operatorname{rank}(C)$. If $k<r$ and $C_{k}=\sum_{i=1}^{k} \sigma_{i} u_{i} v_{i}^{T}$, then

$$
\underset{\operatorname{mank}(D)=k}{\min }\|C-D\|_{2}=\left\|C-C_{k}\right\|_{2}=\sigma_{k+1}
$$

and

$$
\min _{\operatorname{rank}(D)=k}\|C-D\|_{F}=\left\|C-C_{k}\right\|_{F}=\sqrt{\sum_{i=k+1}^{r} \sigma_{i}^{2}} \text {. }
$$

Proof: For proof see Eckart and Young, 1936 and Mirsky, 1960.

(Van Huffel and Vandewalle, 1991, p.31)

Theorem 2.2. (interlacing theorem for singular values) Let $C$ be an $m \times n$ matrix with singular values $\gamma_{1} \geq \cdots \geq \gamma_{\min \{m, n\}}$. Let $D$ be a $p \times q$ submatrix of $C$, with singular values $\delta_{1} \geq \cdots \geq \delta_{\min \{p, q\}}$. Then

$$
\gamma_{i} \geq \delta_{i} \quad \text { for } i=1, \cdots, \min \{p, q\}
$$

and

$$
\delta_{i} \geq \gamma_{i+(m-p)+(n-q)} \quad \text { for } i \leq \min \{p+q-m, p+q-n\}
$$

Proof: For proof see Thompson, 1972.

(Van Huffel and Vandewalle, 1991, p. 32) 
Notice this implies that the largest (respectively, smallest) singular value of a matrix is larger (respectively, smaller) than the largest (respectively, smallest) singular value of any submatrix.

In order to provide a stable and reliable solution, the basic TLS solution will make use of the matrix Singular Value Decomposition (SVD). One approach to finding the TLS solution requires examination of

$$
[A ; b]\left[x^{T} ;-1\right]^{T}=0 \text {. }
$$

If an $x$ can be found such that Equation 2.8 is true, then $x$ is an exact solution to the original system, $A x=b$. Note this implies that $b$ can be written as a linear combination of the columns of $A$. Therefore $\operatorname{rank}([A ; b])=\operatorname{rank}(A)=n$. TLS provides a solution method when $\operatorname{rank}([A ; b])=n+1$. Since $\operatorname{rank}([A ; b])=n+1$ implies no solution to $A x \approx b$ exists, TLS attempts to find the matrix $[\hat{A} ; \hat{b}]$ of rank $n$ which minimizes

$$
\|[A ; b]-[\hat{A} ; \hat{b}]\|_{F} \text { and }\|[A ; b]-[\hat{A} ; \hat{b}]\|_{2} \text {. }
$$

$\operatorname{Rank}(\hat{A})=\operatorname{Rank}([\hat{A} ; \hat{b}])=n$ implies that $\hat{b}$ can be written as a linear combination of the columns of $\hat{A}$ and thus a unique solution to $\hat{A} \hat{x}=\hat{b}$ exists and is given by solving

$$
[\hat{A} ; \hat{b}]\left[\hat{x}^{T}-1\right]^{T}=0
$$

Finding $[\hat{A} ; \hat{b}]$ such that Equation 2.9 is minimized involves use of the SVD and application of the Eckart-Young-Mirsky theorem (Theorem 2.1).

Let the SVD of $[A ; b]$ be defined in the usual way by, $[A ; b]=U \Sigma V^{T}$. If $\sigma_{n+1}=0$, then $\operatorname{rank}([A ; b]) \leq n$. Thus at least one exact solution to Equation 2.8 exists and no TLS approximation is necessary. So consider the case $\sigma_{n+1} \neq 0$. This 
implies $[A ; b]$ has rank $n+1$. Thus the equations are not consistent and no solution to Equation 2.8 exists since $b$ cannot be written as a linear combination of the columns of $A$. In order for a unique solution to exist, the rank of $[A ; b]$ must be reduced to $n$. From Theorem 2.1, the matrix $[\hat{A} ; \hat{b}]=\sum_{i=1}^{n} \sigma_{i} u_{i} v_{i}^{T}=U \hat{\Sigma} V^{T}$ with $\hat{\Sigma}=$ $\operatorname{diag}\left(\sigma_{1}, \cdots, \sigma_{n}, 0\right)$ will be the rank $n$ approximation of $[A ; b]$ which satisfies the TLS problem, and the minimal TLS correction is given by

$$
\sigma_{n+1}=\min _{\operatorname{rank}(\hat{A} ; \hat{i} ;)=n}\|[A ; b]-[\hat{A} ; \hat{b}]\|_{F}=\min _{\operatorname{rank}(\hat{A} ; \hat{b})=n}\|[A ; b]-[\hat{A} ; \hat{b}]\|_{2}
$$

and the TLS correction matrix is then

$$
[\Delta \hat{A} ; \Delta \hat{b}]=[A ; b]-[\hat{A} ; \hat{b}]=\sigma_{n+1} u_{n+1} v_{n+1}^{T} .
$$

Now since $[\hat{A} ; \hat{b}]$ has rank $n$, then $\hat{b}$ can be written as a linear combination of the columns of $\hat{A}$. Thus the set of equations

$$
[\hat{A} ; \hat{b}] z=0
$$

are consistent and the solution is given by the vector $z=v_{n+1} \in N([\hat{A} ; \hat{b}])$. Therefore the TLS solution is found by scaling $v_{n+1}$ until $v_{n+1, n+1}$ is -1 or

$$
\left[\hat{x}^{T} ;-1\right]^{T}=-\frac{1}{v_{n+1, n+1}} v_{n+1}
$$

and

$$
\hat{x}=-\frac{1}{v_{n+1, n+1}}\left[v_{1, n+1}, \cdots, v_{n, n+1}\right]^{T} .
$$

The next theorem gives the conditions for the existence and uniqueness of a TLS solution to $A x \approx b$.

Theorem 2.3. (Existence and Uniqueness of a TLS solution) Suppose the SVD's of $A$ and $[A ; b]$ are given by $A=U^{\prime} \Sigma^{\prime} V^{\prime T}$ and $[A ; b]=U \Sigma V^{T}$. If $\sigma_{n}^{\prime}>\sigma_{n+1}$, then 


$$
[\hat{A} ; \hat{b}]=U \hat{\Sigma} V^{T} \text { and, } \hat{\Sigma}=\operatorname{diag}\left(\sigma_{1}, \cdots, \sigma_{n}, 0\right),
$$

with corresponding TLS correction matrix

$$
[\Delta \hat{A} ; \Delta \hat{b}]=[A ; b]-[\hat{A} ; \hat{b}]=\sigma_{n+1} u_{n+1} v_{n+1}^{T},
$$

solves the TLS problem, Equation 2.1 and Equation 2.2, and

$$
\hat{x}=-\frac{1}{v_{n+1, n+1}}\left[v_{1, n+1}, \cdots, v_{n, n+1}\right]^{T}
$$

exists and is the unique solution to $\hat{A} \hat{x}=\hat{b}$.

Proof: The singular value interlacing theorem 2.2 implies that

$$
\sigma_{1} \geq \sigma_{1}^{\prime} \geq \cdots \geq \sigma_{n} \geq \sigma_{n}^{\prime} \geq \sigma_{n+1} .
$$

The assumption $\sigma_{n}^{\prime}>\sigma_{n+1}$ thus ensures that $\sigma_{n+1}$ is not a repeated singular value of $[A ; b]$. If $[A ; b]^{T}[A ; b]\left[\begin{array}{ll}y & 0\end{array}\right]^{T}=\sigma_{n+1}^{2}\left[\begin{array}{ll}y & 0\end{array}\right]^{T}$ and $0 \neq y \in \mathbb{R}^{n}$, then it clearly follows that $A^{T} A y=\sigma_{n+1}^{2} y$, which is a contradiction since $\sigma_{n}^{\prime 2}$ is the smallest eigenvalue of $A^{T} A$. Thus $N([\hat{A} ; \hat{b}])$ must contain a vector whose $(n+1)$ th component is non-zero. This implies that the TLS problem has a solution. Since $N([\hat{A} ; \hat{b}])$ has dimension one, this solution must be unique. Equations 2.14-2.16 follow straightforwardly from the application of the Eckart-Young-Mirsky Theorem, as shown above.

(Van Huffel and Vandewalle, 1991, pp. 34-35)

\subsubsection{The mixed LS/TLS problem}

In some cases the set of linear equations $A x \approx b$ may include some columns of $A$ which are known exactly; that is, some columns of $A$ may contain error free elements. Thus to ensure the most accurate results in estimating the solution $x$, the modifications to $A$ should require that the error-free columns of $A$ remain unperturbed 
in the estimated matrix $\hat{A}$. This leads to the mixed LS/TLS problem defined as follows:

Definition 2.2. (mixed LS/TLS problem) Given a set of $m$ linear equations in $n$ unknowns $x$

$$
A x \approx b
$$

where

$$
A \in \mathbb{R}^{m \times n}, b \in \mathbb{R}^{m} \text {, and } x \in \mathbb{R}^{n} \text {. }
$$

Partition $A$ and $x$ as

$$
A=\left[A_{1} ; A_{2}\right] \text { and } x=\left[x_{1}^{T} ; x_{2}^{T}\right]^{T}
$$

with

$$
\begin{aligned}
& A_{1} \in \mathbb{R}^{m \times n_{1}}, \\
& A_{2} \in \mathbb{R}^{m \times n_{2}}, \\
& x_{1} \in \mathbb{R}^{n_{1}}, \text { and } \\
& x_{2} \in \mathbb{R}^{n_{2}} .
\end{aligned}
$$

Assume that the columns of $A_{1}$ are error-free and $n=n_{1}+n_{2}$. Then, the mixed LS/TLS problem seeks to

$$
\begin{aligned}
& \underset{\left[\hat{A}_{2} ; \hat{b}\right] \in \mathbf{R}^{\mathbf{m} \times\left(\mathbf{p}_{2}+1\right)}}{\operatorname{minimiz}}\left\|\left[A_{2} ; b\right]-\left[\hat{A}_{2} ; \hat{b}\right]\right\|_{F} \\
& \text { subject to } \hat{b} \in R(\hat{A})=R\left(\left[A_{1} ; \hat{A}_{2}\right]\right) \text {. }
\end{aligned}
$$

Once a minimizing $\left[\hat{A}_{2} ; \hat{b}\right]$ is found, then any $\hat{x}=\left[\hat{x}_{1}^{T} ; \hat{x}_{2}^{T}\right]^{T}$ satisfying

$$
\hat{A} \hat{x}=A_{1} \hat{x}_{1}+\hat{A}_{2} \hat{x}_{2}=\hat{b}
$$

is called a mixed LS/TLS solution and $\left[\Delta \hat{A}_{2} ; \Delta \hat{b}\right]=\left[A_{2} ; b\right]-\left[\hat{A}_{2} ; \hat{b}\right]$ is the corresponding mixed LS/TLS correction matrix. 
(Van Huffel and Vandewalle, 1991, pp. 84-85)

The solution to the mixed LS/TLS problem involves computing the QR factorization of $\left[A_{1} ; A_{2} ; b\right]$ and then solving a basic TLS problem of reduced order. The QR factorization is used since the 2-norm and Frobenius norm are unchanged when the orthogonal transformation $Q$ is applied to $[A ; b]$ and the resulting structure of $R$ allows for the reduction of order of the TLS problem. Let $A$ be partitioned as in Equation 2.18 where $A_{1}$ contains the error free columns of $A$ and is full rank. Next find a QR factorization of $\left[A_{1} ; A_{2} ; b\right]$ such that

$$
Q^{T}\left[A_{1} ; A_{2} ; b\right]=\left[\begin{array}{ccc}
R_{11} & R_{12} & R_{1 b} \\
0_{\left(m-n_{1}\right) \times n_{1}} & R_{22} & R_{2 b}
\end{array}\right]
$$

where

$$
\begin{aligned}
& R_{11} \in \mathbb{R}^{n_{1} \times n_{1}}, \\
& R_{12} \in \mathbb{R}^{n_{1} \times\left(n-n_{1}\right)}, \\
& R_{22} \in \mathbb{R}^{\left(m-n_{1}\right) \times\left(n-n_{1}\right)}, \\
& R_{1 b} \in \mathbb{R}^{n_{1}}, \text { and } \\
& R_{2 b} \in \mathbb{R}^{m-n_{1}} .
\end{aligned}
$$

Now the LS/TLS solution can be computed using $R=Q^{T}\left[A_{1} ; A_{2} ; b\right]$. Compute the solution $\hat{x}_{2}$ to the basic TLS problem $R_{22} x \approx R_{2 b}$. This yields the last $n-n_{1}$ components of the solution vector $\hat{x}$. The first $n_{1}$ elements of $\hat{x}$ are found by solving

$$
R_{11} \hat{x}_{1}=R_{1 b}-R_{12} \hat{x}_{2} \text {. }
$$

The solution, $\hat{x}_{1}$ is simply the LS solution obtained by projecting the reduced observation vector, $b-A_{2} \hat{x}_{2}$, into the range of $A_{1}$ generated by the error free columns of $A$. (Van Huffel and Vandewalle, 1991, p. 85) 
Using a generalization of the Eckart-Young-Mirsky theorem (Theorem 2.1), Golub, Hoffman, and Stewart proved that the above procedure for computing the mixed LS-TLS estimate $\left[\hat{x}_{1}^{T} ; \hat{x}_{2}^{T}\right]^{T}$, finds the best rank $r$ approximation $\left[A_{1} ; \hat{A}_{2} ; \hat{b}\right]$ $(r \leq n)$ to $[A ; b]$ that leaves $A_{1}$ fixed such that

$$
\left\|\left[A_{1} ; A_{2} ; b\right]-\left[A_{1} ; \hat{A}_{2} ; \hat{b}\right]\right\|_{F}=\min _{\text {rank }\left[A_{1} ; \tilde{A}_{2} ; \tilde{b}_{1} \leq r\right.}\left\|\left[A_{1} ; A_{2} ; b\right]-\left[A_{1} ; \tilde{A}_{2} ; \tilde{b}\right]\right\|_{F} .
$$

(Van Huffel and Vandewalle, 1991, p. 86) Theorem 2.4 shows conditions for the existence and uniqueness of the mixed LS/TLS solution.

Theorem 2.4 (Existence and uniqueness of the mixed LS/TLS solution) Consider the mixed LS/TLS problem, Equations 2.17-2.20, and assume that $A_{1}$ has full rank $n_{1}$. Denote by $\sigma^{\prime}$, (respectively, $\sigma$ ) the smallest (respectively, $\left(n_{2}+1\right)$ th) singular value of $R_{22}$ (respectively, $\left[R_{22} ; R_{2 b}\right]$ ) as defined by equation 2.22 , then $\sigma^{\prime}>\sigma$ implies the solution $\hat{x}=\left[\hat{x}_{1}^{T} ; \hat{x}_{2}^{T}\right]^{T}$ of the mixed LS/TLS problem exists and is unique.

Proof: This is a special case of Theorem 3 in Van Huffel and Vandewalle, 1989. (Van Huffel and Vandewalle, 1991, p. 86)

\subsection{Constrained Least Squares (CLS)}

When a set of linear equations, $A x \approx b$, is solved using ordinary least squares, it is sometimes appropriate to restrict the solution vector, $\hat{x}$, to some subset of $\mathbb{R}^{n}$. In this situation, a Constrained Least Squares (CLS) solution method is used. The constraints may be equality or inequality constraints. Section 2.2 considers the equality constrained least squares solution to the overdetermined system. 
Definition 2.3 (equality constrained least squares solution) Let $A \in \mathbb{R}^{m \times n}, B \in$ $\boldsymbol{R}^{p \times n}, b \in \mathbb{R}^{m}, d \in \mathbb{R}^{p}$, and $\operatorname{rank}(B)=p$. Then the equality constrained least squares problem seeks to

$$
\min _{\mathbf{b} \in \mathbf{R}(\mathrm{A})}\|A x-b\|_{2}
$$

subject to

$$
B x=d .
$$

This can be written more compactly as

$$
\min _{\mathbf{B x}=\mathbf{d}}\|x-b\|_{2} \text {. }
$$

The matrix $B$ and vector $d$ are carefully selected to limit the set of possible solution vectors and are specific to each problem. The solution to the equality constrained LS problem is similar to that of the mixed LS/TLS problem of Section 2.1.2 in that a $\mathrm{QR}$ factorization is found and then an unconstrained LS problem of reduced order is solved. Finally, the solution to the reduced order unconstrained LS problem is used to determined the total solution to the constrained LS problem. First, let

$$
Q^{T} B^{T}=\left[\begin{array}{c}
R \\
0_{(n-p) \times p}
\end{array}\right], \quad R \in \mathbb{R}^{p \times p}
$$

be the QR factorization of $B^{T}$ and set

$$
A Q=\left[\begin{array}{ll}
A_{1} & A_{2}
\end{array}\right] \text { and } Q^{T} x=\left[\begin{array}{l}
y \\
z
\end{array}\right]
$$

where 


$$
\begin{aligned}
& A_{1} \in \mathbb{R}^{m \times p}, \\
& A_{2} \in \mathbb{R}^{m \times(n-p)}, \\
& y \in \mathbb{R}^{p}, \text { and } \\
& z \in \mathbb{R}^{n-p} .
\end{aligned}
$$

Then, with these transformations, Equation 2.27 becomes

$$
\min _{\mathrm{R}_{\mathrm{T}}=\mathrm{d}}\left\|A_{1} y+A_{2} z-b\right\|_{2} \text {. }
$$

Now $y$ is determined from the constraint equation $R^{T} y=d$ and the vector $z$ is obtained by solving the unconstrained LS problem

$$
\min _{\mathrm{z}}\left\|A_{2} z+\left(b-A_{1} y\right)\right\|_{2} \text {. }
$$

Finally, $y$ and $z$ can be substituted into Equation 2.29 so that

$$
x=Q\left[\begin{array}{l}
y \\
z
\end{array}\right]
$$

solves the constrained LS problem defined in Equations 2.25-2.26. (Golub and Van Loan, 1989, pp. 561-567) 


\section{Chapter 3: Transfer Function Determination Code (TFDC)}

The Transfer Function Determination Code (TFDC) is a system identification algorithm for determining the input-output transfer function of a single-input, singleoutput (SISO) discrete-time system. Using frequency response data as input, TFDC computes the numerator and denominator coefficients of the discrete-time transfer function, $G(z)$, given by

$$
G(z)=\frac{a(z)}{b(z)}=\frac{a_{n} z^{n}+a_{n-1} z^{n-1}+\cdots+a_{1} z+a_{0}}{b_{n} z^{n}+b_{n-1} z^{n-1}+\cdots+b_{1} z+1} .
$$

To find the coefficients, TFDC performs three steps. First, the user selects the frequency points to be used in the identification process. Next, a system of linear equations is formed from the selected frequency points and complex frequency response data. Finally, a solution is found to the linear system and the coefficients of the identified transfer function are determined from this solution.

Section 3.1 derives the linear system that is solved in TFDC. Section 3.2 is a brief discussion of the results and problems in using an ordinary Least Squares (LS) solution to the system of equations. TFDC code modifications are presented in Section 3.3. Section 3.3.1 discusses the speed improvements. Section 3.3.2 derives the constraint equations used to insure the symmetry of the poles and zeros required of the rational function identified by TFDC when applied to power spectral density estimates. The algorithms used for the TLS, LS/TLS, and CLS solution methods are given in Section 3.3.3. 


\subsection{Derivation of TFDC Equations}

Given $N$ complex numbers

$$
G\left(e^{j \omega_{i} T}\right)=x_{i}+j y_{i}, \quad i=1, \cdots, N,
$$

representing SISO frequency response data obtained from a discrete time system, where $T$ is the sampling period, it is desired to identify an $n$th order transfer function describing the system. This transfer function can be assumed to have the form of Equation 3.1. Considering Equation 3.1, the frequency response at each frequency point, $\omega_{i}$, of this transfer function is given, by setting $z=e^{j \omega_{i} T}$, as

$$
G\left(e^{j \omega_{i} T}\right)=\frac{a\left(e^{j \omega_{i} T}\right)}{b\left(e^{j \omega_{i} T}\right)}=\frac{a_{n} e^{j n \omega_{i} T}+a_{n-1} e^{j(n-1) \omega_{i} T}+\cdots+a_{1} e^{j \omega_{i} T}+a_{0}}{b_{n} e^{j n \omega_{i} T}+b_{n-1} e^{j(n-1) \omega_{i} T}+\cdots+b_{1} e^{j \omega_{i} T}+1}
$$

This is the frequency response of the assumed transfer function at $\omega_{i}$. Substituting

$$
\begin{aligned}
& e^{j k \omega_{i} T}=\cos \left(k \omega_{i} T\right)+j \sin \left(k \omega_{i} T\right) \text { into Equation } 3.3 \text { gives } \\
& \qquad a\left(e^{j \omega_{i} T}\right)=\sum_{k=0}^{n} a_{k} \cos \left(k \omega_{i} T\right)+j \sum_{k=0}^{n} a_{k} \sin \left(k \omega_{i} T\right)
\end{aligned}
$$

and

$$
b\left(e^{j \omega_{i} T}\right)=\sum_{k=1}^{n} b_{k} \cos \left(k \omega_{i} T\right)+j \sum_{k=1}^{n} b_{k} \sin \left(k \omega_{i} T\right)+1
$$

Rearranging Equation 3.3 for the $i$ th frequency point gives

$$
a\left(e^{j \omega_{i} T}\right)=G\left(e^{j \omega_{i} T}\right) b\left(e^{j \omega_{i} T}\right) .
$$

Substituting Equations 3.2, 3.4, and 3.5 into Equation 3.6 gives

$$
\begin{gathered}
\sum_{k=0}^{n} a_{k} \cos \left(k \omega_{i} T\right)+j \sum_{k=0}^{n} a_{k} \sin \left(k \omega_{i} T\right)= \\
\left(x_{i}+j y_{i}\right)\left(\sum_{k=1}^{n} b_{k} \cos \left(k \omega_{i} T\right)+j \sum_{k=1}^{n} b_{k} \sin \left(k \omega_{i} T\right)+1\right)
\end{gathered}
$$


Carrying out the multiplication and equating real and imaginary parts yields two linear equations in $2 n+1$ unknowns:

$$
x_{i}=\sum_{k=0}^{n} a_{k} \cos \left(k \omega_{i} T\right)+\sum_{k=1}^{n} b_{k}\left(y_{i} \sin \left(k \omega_{i} T\right)-x_{i} \cos \left(k \omega_{i} T\right)\right)
$$

and

$$
y_{i}=\sum_{k=0}^{n} a_{k} \sin \left(k \omega_{i} T\right)+\sum_{k=1}^{n} b_{k}\left(-x x_{i} \sin \left(k \omega_{i} T\right)-y_{i} \cos \left(k \omega_{i} T\right)\right)
$$

Define $\theta, \mathrm{h}_{\mathrm{iR}}$, and $\mathrm{h}_{\mathrm{iI}}$ as

$$
\begin{aligned}
& \theta=\left[\begin{array}{llllllll}
a_{0} & a_{1} & \cdots & a_{n} & b_{1} & b_{2} & \cdots & b_{n}
\end{array}\right]^{T} \\
& h_{i R}=\left[\begin{array}{llllll}
1 & \cos \left(\omega_{i} T\right) & \cos \left(2 \omega_{i} T\right) & \cdots & \cos \left(n \omega_{i} T\right) & y_{i} \sin \left(\omega_{i} T\right)-x_{i} \cos \left(\omega_{i} T\right)
\end{array}\right. \\
& \left.y_{i} \sin \left(2 \omega_{i} T\right)-x_{i} \cos \left(2 \omega_{i} T\right) \quad \cdots \quad y_{i} \sin \left(n \omega_{i} T\right)-x_{i} \cos \left(n \omega_{i} T\right)\right]^{T} \\
& \begin{aligned}
h_{i l}= & {\left[\begin{array}{llllll}
0 & \sin \left(\omega_{i} T\right) & \sin \left(2 \omega_{i} T\right) & \cdots & \sin \left(n \omega_{i} T\right) & -x_{i} \sin \left(\omega_{i} T\right)-y_{i} \cos \left(\omega_{i} T\right) \\
& -x_{i} \sin \left(2 \omega_{i} T\right)-y_{i} \cos \left(2 \omega_{i} T\right) & \cdots & -x_{i} \sin \left(n \omega_{i} T\right)-y_{i} \cos \left(n \omega_{i} T\right)
\end{array}\right]^{T} . }
\end{aligned}
\end{aligned}
$$

Then the linear system

$$
H \theta \approx u
$$

where

$$
\begin{aligned}
H & =\left[\begin{array}{llllllll}
h_{1 R} & h_{2 R} & \cdots & h_{N R} & h_{1 I} & h_{2 I} & \cdots & h_{N I}
\end{array}\right]^{T} \text { and } \\
u & =\left[\begin{array}{llllllll}
x_{1} & x_{2} & \cdots & x_{N} & y_{1} & y_{2} & \cdots & y_{N}
\end{array}\right]^{T}
\end{aligned}
$$

describes the relationship between the transfer function coefficients and the experimental frequency response data points. In general, this system will be overdetermined and the equations will be inconsistent. This implies a least squares solution, i.e. find $\theta$ to minimize $\|H \theta-u\|_{2}$, to the system should be used. Notice that uncertainty is introduced into Equation 3.10 by the noisy test data, $x_{i}^{\prime}$ 's and $y_{i}^{\prime}$ 's. The 
right hand side vector, $u$, contains uncertainty in every component. The coefficient matrix, $H$, contains uncertainty in all elements containing $x_{i}$ 's and $y_{i}$ 's; i.e., the last $n$ columns of $H$. The solution to Equation 3.10 is the heart of the TFDC algorithm. Increasing the accuracy of the solution to Equation 3.10 requires a solution method that accounts for the uncertainty in both $H$ and $u$.

\subsection{Ordinary Least Squares (LS) Solution and the TFDC Algorithm}

TFDC was originally implemented using an ordinary Least Squares (LS) solution to Equation 3.10. This is not the best solution method for this system of equations due to uncertainty in the $H$ matrix. The ordinary LS solution to a set of linear equations is based on finding the nearest projection, as measured by the 2norm, of the right hand side vector to the range space of the coefficient matrix; such that, the set of equations is consistent, i.e. applying the transformation $H\left(H^{T} H\right)^{-1} H^{T}$ to the linear system, $H \theta \approx u$, gives

$$
H\left(H^{T} H\right)^{-1} H^{T} H x=H x=H\left(H^{T} H\right)^{-1} H^{T} u
$$

and thus $\hat{u} \in R(H)$ since

$$
\hat{x}=\left(H^{T} H\right)^{-1} H^{T} u
$$

solves Equation 3.11 where $H\left(H^{T} H\right)^{-1} H^{T}$ is the orthogonal projector of $u$ onto $\mathrm{R}(H)$. Thus in the ordinary LS solution, only the right hand side vector is considered to contain uncertainty since this is the only data that is modified in the LS solution algorithm. The coefficient matrix is not the modified. Since, in the case of TFDC, the coefficient matrix also contains uncertainty in nearly half of its elements, the TLS 
solution method is more suited. The TLS solution modifies both the coefficient matrix and the right hand side vector to account for uncertainty in both $H$ and $u$.

TFDC with the ordinary LS solution produced poor results at first, mostly due to uncertainty in the coefficient matrix. To improve the accuracy of the LS solution, a weighting matrix was added to the linear system in TFDC. Medina (1991) discusses three weighting methods which were implemented in TFDC. These three weighting methods were used in combination to improve the accuracy of the identified system.

Also, according to Medina (1991), the first LS solution at times would miss important features of the frequency response, particularly lightly damped modes. Therefore, the TFDC algorithm was modified to iterate on the LS solution. At each iteration the weights and right hand side vector were modified and then another LS solution was calculated. This also improved the accuracy of the solution. (Medina, 1991)

With all these modifications and much user interaction, acceptable transfer functions have been identified from experimental data, but at the expense of an increase in the complexity of the code and an increase in the time spent by the user in identifying the system models. The significance of this thesis is that the implementation of the TLS, LS/TLS, and CLS solution methods improves the accuracy of the identified transfer function with the added benefit of decreased code complexity. Also, decreased execution times have been achieved via code speed improvements and the elimination of iteration inside the algorithm. 


\subsection{The TFDC Code (Usage and Modifications)}

The TFDC algorithm is currently implemented in a Matlab $\mathrm{m}$-file as a user defined function. It is called from the Matlab command line like any other Matlab function. The outputs of TFDC are two vectors, num and den, which contain, respectively, the numerator and denominator coefficients of the identified system transfer function. The input arguments are

$\mathbf{p}$, the input vector containing the complex data of the frequency response to be identified,

norder, the assumed order of the system to be identified, $\mathbf{w}$, the frequency vector, in $\mathrm{rad} / \mathrm{sec}$, corresponding to the data points in the input vector, $\mathbf{p}$,

fs, the sampling frequency, in $\mathrm{Hz}$, of the discrete system,

npts, the number of linear and logarithmically spaced points to be used in the initial frequency range specified by the user (the points actually used in the identification process will be the actual points in $w$ which are closest to these points),

method, a character string, " $t$ ", "l", "c", or " $s$ " which specifies the method, TLS, LS/TLS, CLS, or LS, respectively, to be using in solving the linear system, and

tfname, a character string that will be used as the title for output plots from TFDC.

At the beginning of TFDC, the user must follow the prompts in order to graphically select the frequency points to be used in the identification process. The user first specifies an initial range of frequencies to be used. Then options are given to allow selection of additional points from the graph or from an expanded plot. After the frequency points have been selected, TFDC identifies the numerator and 
denominator coefficients of Equation 3.1 for the transfer function order specified by

norder. Next, TFDC displays a plot comparing the frequency response of the identified transfer function with the actual data. Finally, TFDC shows a series of error plots and the pole-zero constellation of the identified transfer function. The error plots are the magnitude error, phase error, and absolute error at each frequency point. Also included on the pole-zero constellation plot are the number of unstable poles, if any, in the identified transfer function.

\subsubsection{Speed Improvement}

Two revisions of the code were implemented to increase the execution speed. The first modification was changing the frequency selection algorithm. The TFDC code first allows the user to select the frequency range for which to identify a transfer function. Then, in this frequency range, a vector, wuse, of linearly and logarithmically spaced frequency points is created. Next, the frequencies included in the actual data which are close to those in wuse are selected to be used in the identification process. This selection was previously done by an algorithm which sorted the actual data points in $\mathbf{p}$ and $\mathbf{w}$. For this thesis, the selection process was modified so that the indices of the vectors were sorted and then the data was extracted from $\mathbf{p}$ and $\mathbf{w}$ in the required order. The intent of this modification was to increase the speed of the sorting algorithm by sorting the four byte integer index numbers instead of sorting the eight byte data elements, but this change decreased the sort time only slightly as shown in Table 3.1. The data in Table 3.1 was obtained by executing TFDC with a 
fixed $\mathbf{p}$ and $\mathbf{w}$ and varying the npts argument. The time required was determined using the Matlab command "etime".

\begin{tabular}{|c|c|c||}
\hline Points Selected & $\begin{array}{c}\text { Time by sorting data } \\
\text { (seconds) }\end{array}$ & $\begin{array}{c}\text { Time by sorting in- } \\
\text { dexes (seconds) }\end{array}$ \\
\hline 92 & 16.3742 & 15.9096 \\
\hline 110 & 16.9874 & 16.8329 \\
\hline 127 & 17.2868 & 17.5807 \\
\hline 143 & 18.0653 & 17.9154 \\
\hline 162 & 18.6202 & 18.2374 \\
\hline 179 & 19.8216 & 19.0676 \\
\hline 338 & 25.8612 & 25.1098 \\
\hline 489 & 31.5214 & 31.5790 \\
\hline 642 & 38.4305 & 36.7201 \\
\hline 775 & 45.6765 & 44.0361 \\
\hline 904 & 53.2432 & 50.2749 \\
\hline 1033 & 61.1354 & 56.6996 \\
\hline 1171 & 69.1428 & 62.3444 \\
\hline 1290 & 77.2395 & 73.3432 \\
\hline 1415 & 82.3403 & 79.8107 \\
\hline
\end{tabular}

Table 3.1: Comparison of Frequency Selection Times

The second modification involves the code segment which builds the set of linear equations from the input data. After the desired frequencies are selected, TFDC must build the linear system, $H \theta \approx u$, from the frequency response at those frequencies. The right hand side vector, $u$, is easily formed by concatenating the vector of imaginary parts of the complex frequency response onto the vector of the 
real part of the response. A significant amount of execution time, though, is spent in forming the $H$ matrix. Three methods have been tried in forming this matrix.

Method 1. Originally $H$ was formed using two levels of nested "for" loops. Since the Matlab programming language is an interpreted language, "for" loops have long execution times due to the fact that each command in the loop must be reinterpreted at each loop iteration. This method is easy to read and understand since it has the same structure used in most high level programming languages, but it is very slow in comparison with Methods 2 and 3. The Matlab manual suggests, if possible, replacing "for" loops with "vectorized" calculations to increase execution speed. (The Mathworks, Inc., 1990, p. 2-90,2-91) This has been implemented in Method 2.

Method 2. This method eliminates the use of looping and forms $H$ with only vector and matrix operations. This is accomplished by forming the required diagonal matrices and pre- and post-multiplying the data elements by these diagonal matrices. This method has the fastest execution time for small sets of data, but, as the size of the data set increases, the size of the diagonal matrices increases accordingly. This makes the memory requirements for this method large and thus virtual memory is employed when a large number of data points are used. When the data set is large enough that virtual memory is used, the execution time increases drastically since data has to be swapped to and from the disk drive. This method has been discarded for two reasons. First, the accuracy of the solution to the linear equations increases as more data points are used, therefore, a large enough data set is generally used so that virtual memory is required. Second, when a small enough data set is used so that 
virtual memory is not required by this method, the difference in execution times for Methods 2 and 3 is insignificant.

Method 3. This is the fastest method without the need for a large amount of memory overhead. This method, in effect, "vectorizes" the inner loop from Method 1, and leaves one "for" loop around these vectorized calculations. Thus each row of the coefficient matrix is formed by vector and matrix calculations and then using the "for" loop all the rows are concatenated one over the other to form the $H$ matrix.

Both Method 2 and 3 execute significantly faster than Method 1. Method 3 was chosen as the best method since it has the fastest execution time when a large number of data points are used without additional memory overhead. Table 3.2 compares the times required by each of the three methods in building the set of linear equations to be solved. This data was compiled using the same arguments for TFDC described above for the data in Table 3.1.

Thus as seen from the data in Table 3.1 and especially in Table 3.2 the execution time of the TFDC algorithm has been decreased without sacrificing the amount of memory required by the algorithm.

\subsubsection{Constraint Equations for the CLS Solution Method}

TFDC, when applied to power spectral density (psd) estimates of the system output sequence, identifies the rational function $G\left(e^{-j \omega T}\right) G\left(e^{j \omega T}\right)$. Let $z=e^{j \omega T}$ and

$$
G(z) G\left(z^{-1}\right) \Delta \frac{Q(z) Q\left(z^{-1}\right)}{R(z) R\left(z^{-1}\right)}
$$

By applying the bilinear transformation, $z=\frac{1+w}{1-w}$, to Equation 3.13 


\begin{tabular}{|c|c|c|c||}
\hline $\begin{array}{c}\text { Points } \\
\text { Selected }\end{array}$ & $\begin{array}{c}\text { Time using method } \\
1 \text { (seconds) }\end{array}$ & $\begin{array}{c}\text { Time using Method } \\
2 \text { (seconds) }\end{array}$ & $\begin{array}{c}\text { Time using method } \\
3 \text { (seconds) }\end{array}$ \\
\hline 92 & 2.5880 & 0.1233 & 0.6335 \\
\hline 110 & 3.1613 & 0.2347 & 0.7477 \\
\hline 127 & 3.6774 & 0.2466 & 0.8527 \\
\hline 143 & 4.1208 & 0.3059 & 0.9512 \\
\hline 162 & 4.6502 & 0.4422 & 1.0545 \\
\hline 179 & 5.1441 & 0.4772 & 1.1504 \\
\hline 338 & 10.7160 & 2.2359 & 2.2076 \\
\hline 489 & 16.5440 & 4.2563 & 3.2085 \\
\hline 642 & 23.4703 & 7.5058 & 4.1997 \\
\hline 775 & 29.5096 & 10.4893 & 5.0368 \\
\hline 904 & 36.5780 & 16.9571 & 5.8507 \\
\hline 1033 & 42.7861 & 33.5348 & 6.6777 \\
\hline 1171 & 51.1629 & 74.1220 & 7.6248 \\
\hline 1290 & 58.3368 & $>240$ & 8.1547 \\
\hline 1415 & 68.7645 & $>240$ & 9.1367 \\
\hline \hline
\end{tabular}

Table 3.2: Comparison of Times to Build Linear Equations

$$
G(w) G(-w)=\frac{Q(w) Q(-w)}{R(w) R(-w)}
$$

can be considered. The roots of $Q(w) Q(-w)$ and the roots of $R(w) R(-w)$ occur with quadrantal symmetry. (Lam, 1979, p. 53) Thus the poles and zeros of $G(w) G(-w)$ should occur with quadrantal symmetry. The CLS solution method was implemented so that this constraint on the poles and zeros could be applied when the input to TFDC is psd data. 
The quadrantal symmetry of the poles and zeros can be achieved by constraining the odd power coefficients of $w$ to be equal to zero in both $Q(w) Q(-w)$ and $R(w) R(-w)$. But since TFDC identifies the coefficients in the discrete time domain, an algorithm was developed to relate these constraints on the w-plane coefficients to the z-plane coefficients. The algorithm implemented in TFDC is described in Section 3.3.3. Thus the constraint equations are determined by this algorithm by following the same algebraic steps used to calculate the constraints by hand.

This algorithm is best illustrated by an example. Assume a second order rational function is to be identified by TFDC. Then the assumed form of the transfer function is given by

$$
G(z)=\frac{a_{2} z^{2}+a_{1} z+a_{0}}{b_{2} z^{2}+b_{1} z+1} .
$$

Then applying the bilinear transformation, $z=\frac{1+w}{1-w}$, and multiplying by $\left(\frac{1-w}{1-w}\right)^{2}$ yields

$$
G(w)=\frac{a_{2}(1+w)^{2}+a_{1}(1+w)(1-w)+a_{0}(1-w)^{2}}{b_{2}(1+w)^{2}+b_{1}(1+w)(1-w)+(1-w)^{2}} .
$$

Now expanding the products involving $w$ and collecting terms gives

$$
G(w)=\frac{\left(a_{2}-a_{1}+a_{0}\right) w^{2}+\left(2 a_{2}-2 a_{0}\right) w+\left(a_{2}+a_{1}+a_{0}\right)}{\left(b_{2}-b_{1}+1\right) w^{2}+\left(2 b_{2}-2\right) w+\left(b_{2}+b_{1}+1\right)} .
$$

Then the constraint equations on the z-plane coefficients can be determined directly from Equation 3.17 by setting the coefficients of the $w^{1}$ terms in the numerator and denominator equal to zero. For this example the constraint equations would then be given by

$$
2 a_{2}-2 a_{0}=0 \text { and } 2 b_{2}-2=0
$$


Note that the number of constraint equations will be equal to the assumed order of the rational function to be identified by TFDC. Half the constraints will come from constraining quadrantal symmetry on the zeros and half from constraining quadrantal symmetry on the poles. Algorithm 3.4 finds a linear system $B x=d$ describing the constraints using the arbitrary model order entered as a parameter to TFDC.

\subsubsection{Algorithms Used in TLS, LS/TLS, and CLS solutions}

The final modification to the TFDC algorithm is the addition of the TLS, LS/TLS, and CLS solution methods and allowing the user to choose the solution method used. A command line argument was added to allow the user to select which algorithm is used in solving the linear system formed in TFDC. The necessary code segments to perform each of these solutions were added, and the user must specify by the argument, method, which solution method will be used by TFDC. Three companion m-files were added to the TFDC package. An m-file called "tlssolvr.m" was written to perform the TLS solution and is called by TFDC when this solution is required. Two m-files, "buildco.m" and "coeff.m", were written to form the linear system describing the constraints used in the CLS solution. The algorithms used for each method are given below.

Algorithm 3.1. (Basic TLS Solution Algorithm) The algorithm for the basic TLS solution to $A x \approx b$ is implemented in the m-file, "tlssolvr.m".

Given: $A \in \mathbb{R}^{m \times n}$ and $b \in \mathbb{R}^{m}$.

Step 1: Compute the SVD of $[A ; b]=U \Sigma V^{T}$.

Step 2: If $v_{n+1, n+1} \neq 0$, then $\hat{x}=-\frac{1}{v_{n+1, n+1}}\left[v_{1, n+1}, \cdots, v_{n, n+1}\right]^{T}$. 
END

Algorithm 3.2. (LS/TLS Solution Algorithm) The algorithm for the LS/TLS solution is as follows

Given: $A \in \mathbb{R}^{m \times n}$ with the first $n_{1}$ columns of $A$ being error free and $b \in \mathbb{R}^{m}$.

Step 1: Find the QR factorization of $[A ; b]$ as given in Equation 2.22 .

Step 2: Find the basic TLS solution, $\hat{x}_{2}$, to $R_{22} x_{2} \approx R_{2 b}$ using Algorithm 3.1.

Step 3: Find the solution to $R_{11} \hat{x}_{1}=R_{1 b}-R_{12} \hat{x}_{2}$ by $\hat{x}_{1}=R_{11}^{-1}\left(R_{1 b}-R_{12} \hat{x}_{2}\right)$.

Step 4: The LS/TLS solution, $\hat{x}$, is given by $\hat{x}=\left[\hat{x}_{1}^{T} ; \hat{x}_{2}^{T}\right]^{T}$. END

Algorithm 3.3. (CLS Solution Algorithm) The algorithm for finding the CLS solution to the overdetermined set of equations follows.

Given: $A \in \mathbb{R}^{m \times n}, B \in \mathbb{R}^{p \times n}, b \in \mathbb{R}^{m}$, and $d \in \mathbb{R}^{p}$ with $\operatorname{rank}(A)=n$ and $\operatorname{rank}(B)=p$.

Step 1: Find the QR factorization of $B^{T} ; B^{T}=Q R$.

Step 2: Solve the linear system $R(1: p, 1: p)^{T} y=d$.

Step 3: Form the product $A=A Q$.

Step 4: Find the LS solution, $z$, so that $\|A(:, p+1: n) z-(b-A(:, 1: p) y)\|_{2}$ is minimized.

Step 5: The CLS solution, $x$, is given by $x=Q(:, 1: p) y+Q(:, p+1: n) z$. $\underline{\text { END }}$

Algorithm 3.4. (forming CLS constraint equations) This algorithm forms the constraint equations used in finding the CLS solution. 
Given: $n$ the assumed model order.

Step 1: Let $p=0$.

Step 2: Form the binomial expansions of $(1+s)^{p}$ and $(1-s)^{n-p}$. Let $a$ (respectively, $b)$ be the vector of coefficients of $(1+s)^{p}$ (respectively $\left.(1-s)^{n-p}\right)$

Step 3: Find the coefficients of the product $(1+s)^{p}(1-s)^{n-p}$, by out $(p+1,:)=$ $a * b$, where * denotes discrete convolution.

Step 4: $p=p+1$

Step 5: If $p<n$, repeat Steps 2 through 4.

Step 6: Let $p=2$.

Step 7: $o u t=o u t^{\mathrm{T}}$.

Step 8: Let $\operatorname{constr}(p / 2,:)=\operatorname{out}(p,:)$, and $p=p+2$.

Step 9: If $p<n$, repeat Step 8 .

Step 10: Form the coefficient matrix and right hand side vector of the constraint system, $B x=d$, by the following

$$
B=\left[\begin{array}{cc}
\text { constr } & 0_{(n / 2) \times n} \\
0_{(n / 2) \times(n+1)} & \text { constr(:,2:n+1) }
\end{array}\right], d=\left[\begin{array}{c}
0_{(n / 2) \times 1} \\
- \text { constr(:,1) }
\end{array}\right] .
$$

\section{$\underline{\text { END }}$}




\section{Chapter 4: System Identification Using Modified TFDC}

Chapter 4 presents the results of using the modified TFDC code in system identification. The modified code was tested on two sets of frequency response data. One data set is from a linear simulation of a constructed example system and the other data set is from a system at the NASA Marshall Space Flight Center in Huntsville, Alabama. Section 4.1 describes the systems and the methods of obtaining the data used as input to TFDC. The simulation used to obtain time data from the constructed system is described in Section 4.2 and the method used to generate frequency domain data from time domain data is discussed in Section 4.3. Section 4.4 presents the results of identification of the constructed system with each of the solution methods above. Also, a comparison is made of the TLS and LS/TLS methods. The same discussion for the NASA data is contained in Section 4.5.

\subsection{Description of Data Used to Test the Modified TFDC Code}

The modified TFDC code was tested on two sets of data. The first set of data was derived by a linear simulation of a constructed example system. This system is a two input, two output system with two lightly damped modes. It has been used in testing a number of system identification codes at Ohio University. The example system is derived from the following continuous time transfer function matrix

$$
G(s)=\frac{\left[\begin{array}{cc}
.5 & 1 \\
.25 & .5
\end{array}\right]}{s^{2}+.01 s+1}+\frac{\left[\begin{array}{cc}
.3 & -.03 \\
-.6 & .06
\end{array}\right]}{s^{2}+.02 s+4}
$$

A state-space realization of $G(s)$ above is given by 


$$
\begin{gathered}
A_{c}=\left[\begin{array}{cccc}
0 & 1.0 & 0 & 0 \\
-1.0 & -0.01 & 0 & 0 \\
0 & 0 & 0 & 1.0 \\
0 & 0 & -4.0 & -0.02
\end{array}\right] \quad B_{c}=\left[\begin{array}{cc}
0 & 0 \\
1 & 2 \\
0 & 0 \\
1 & -0.1
\end{array}\right] \\
C_{c}=\left[\begin{array}{cccc}
0.5 & 0 & 0.3 & 0 \\
0.25 & 0 & -0.6 & 0
\end{array}\right] \quad D_{c}=\left[\begin{array}{ll}
0 & 0 \\
0 & 0
\end{array}\right] .
\end{gathered}
$$

This continuous system, $\left(A_{c}, B_{c}, C_{c}, D_{c}\right)$, can be discretized to $\left(A_{d}, B_{d}, C_{d}, D_{d}\right)$ by the Matlab function C2D (with the assumption that the system inputs are sampled and held by a zero-order hold with a sampling time of .3 seconds). The discretized realization is then given by

$$
\begin{gathered}
A_{d}=\left[\begin{array}{cccc}
0.9554 & 0.2951 & 0 & 0 \\
-0.2951 & 0.9524 & 0 & 0 \\
0 & 0 & 0.8257 & 0.2815 \\
0 & 0 & -1.1259 & 0.8201
\end{array}\right] \quad B_{d}=\left[\begin{array}{ccc}
0.0446 & 0.0892 \\
0.2951 & 0.5902 \\
0.0436 & -0.0044 \\
0.2815 & -0.0281
\end{array}\right] \\
C_{d}=\left[\begin{array}{cccc}
0.5 & 0 & 0.3 & 0 \\
0.25 & 0 & -0.6 & 0
\end{array}\right] \quad D_{d}=\left[\begin{array}{ll}
0 & 0 \\
0 & 0
\end{array}\right] .
\end{gathered}
$$

The first mode has a natural frequency of $\omega_{n_{1}}=1 \mathrm{rad} / \mathrm{sec}$. and damping ratio $\zeta_{1}=$ .005. The second mode has a natural frequency of $\omega_{n_{2}}=2 \mathrm{rad} / \mathrm{sec}$. and damping ratio $\zeta_{2}=.005$. The analytical frequency responses for each input, output pair are found in Figure 4.1 - Figure 4.4.

In identifying an actual system, usually time domain data will be available. Thus, a linear simulation of the constructed system, $\left(A_{d}, B_{d}, C_{d}, D_{d}\right)$, must be done in order to obtain time domain data for the system. In building the simulation and selecting the parameters to be used, the time-domain characteristics of the system must be considered. The sampling time, $T$, must be small enough so that the 

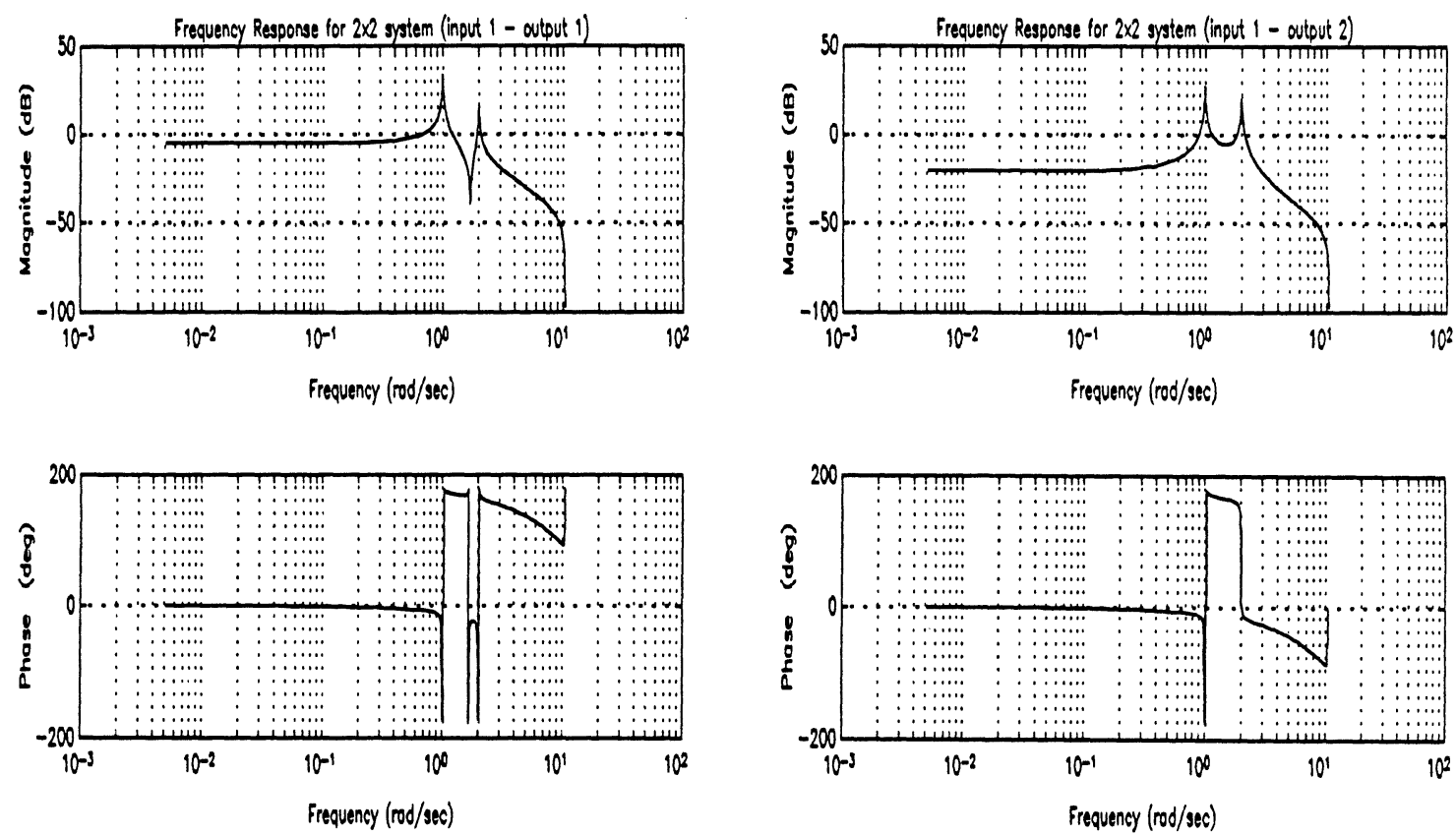

Figure 4.1: Analytical Frequency Response for the Constructed System, $\mathrm{G}_{11}(\mathrm{z})$.
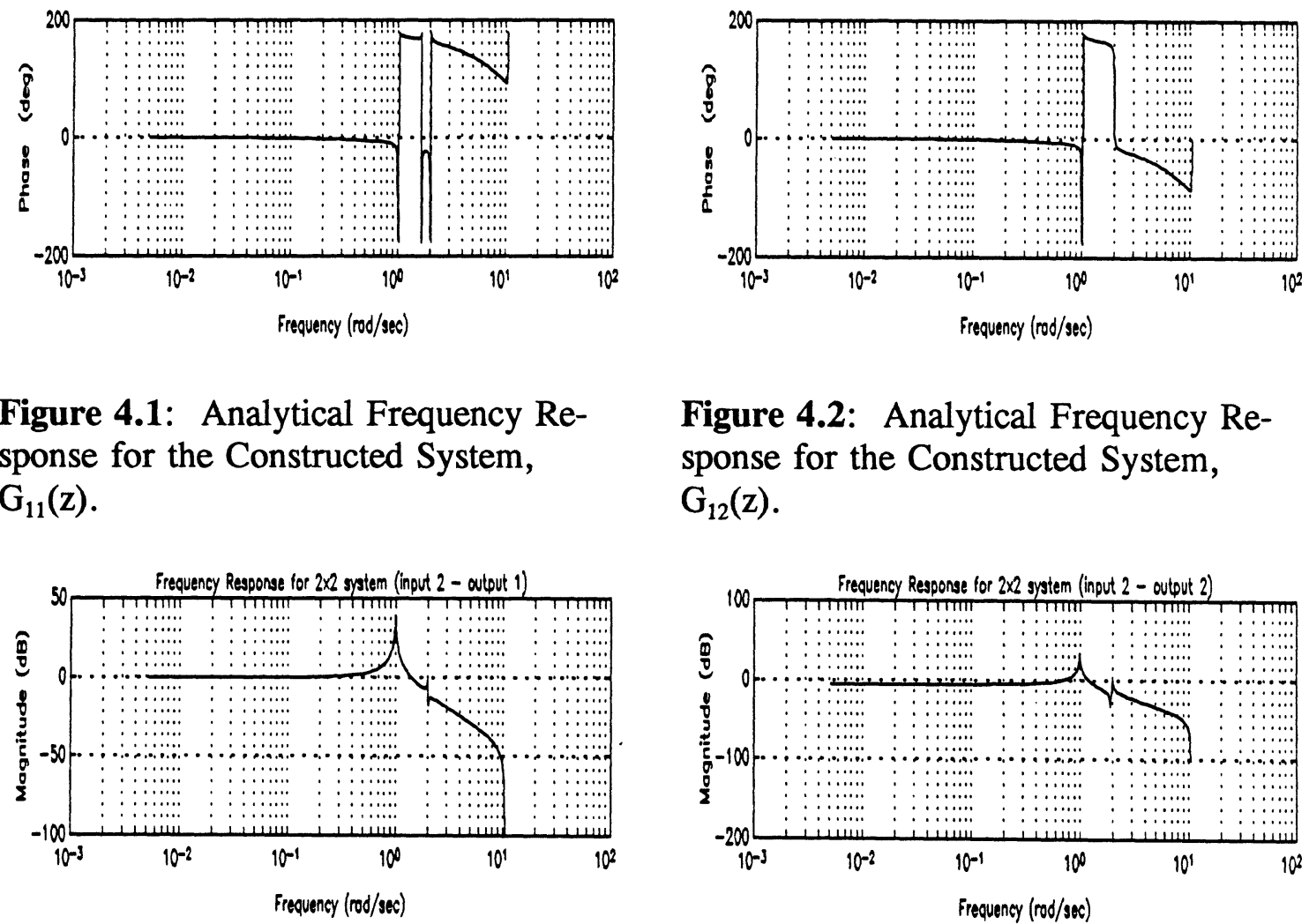

Figure 4.2: Analytical Frequency Response for the Constructed System, $\mathrm{G}_{12}(\mathrm{z})$.
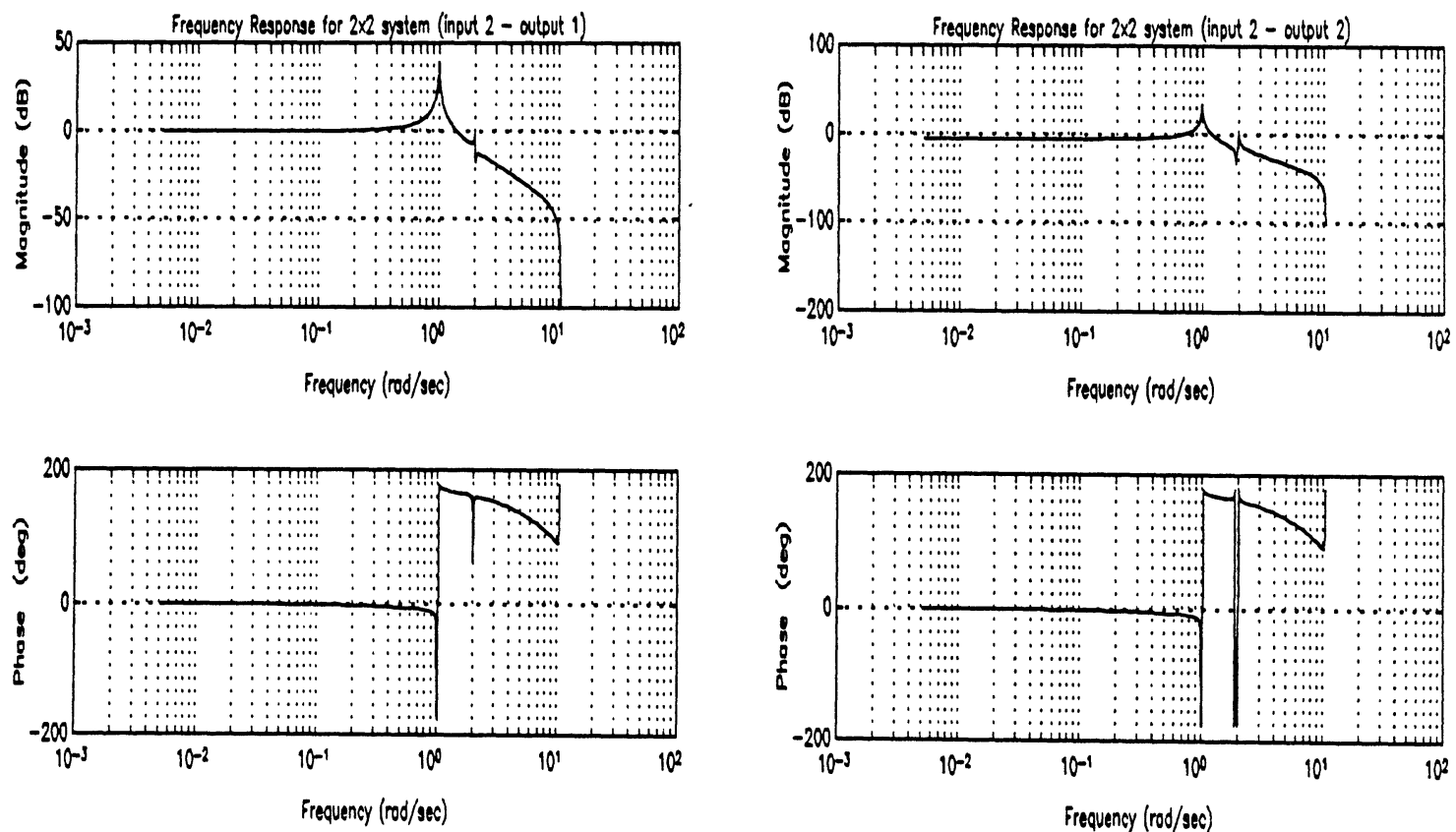

Figure 4.3: Analytical Frequency Response for the Constructed System, $\mathrm{G}_{21}(\mathrm{z})$.

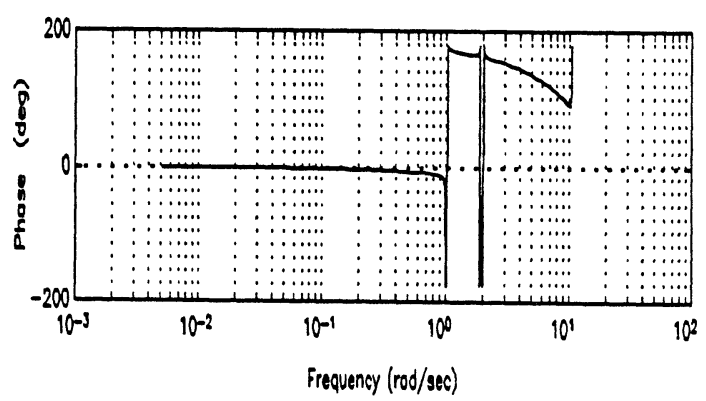

Figure 4.4: Analytical Frequency Response for the Constructed System, $\mathrm{G}_{22}(\mathrm{z})$. 
sampling frequency is above the Nyquist rate for the system. The highest natural frequency for the constructed system is $.318 \mathrm{~Hz}$. Thus a sampling time of $T=.3$ seconds (i.e. $f_{s}=3.33 \mathrm{~Hz}$.) was selected. Also, a long enough data record must be collected so that the time span of the simulation is much longer than the longest time constant of the system. The slowest natural frequency of the system is $.159 \mathrm{~Hz}$.

which corresponds to a time constant of $\frac{1}{\zeta_{1} \omega_{n_{1}}}=200$ seconds. Since steady state is usually considered to be four times the longest time constant, at least 800 seconds of data should be collected. With a sampling time of .3 seconds this requires approximately 2700 data points from the simulation. This simulation is described in the next section.

The modified TFDC algorithm was also applied to data obtained from the NASA Large Space Structure Ground Test Facility (LSS GTF) at the Marshall Space Flight Center in Huntsville, Alabama. This facility is shown in Figure 4.5. TFDC was applied to data from the AGS-BG subsystem (devices 3 and 4 of Figure 4.5) described by Medina (1991).

\subsection{Using Matlab's SIMULAB Toolbox for the Linear Simulation}

The simulation was constructed and executed using Matlab's SIMULAB toolbox. SIMULAB is a graphic oriented interface to the Matlab environment which allows the user to select elements and compound blocks from component libraries and build a numerical simulation via a block diagram of the system. Figure 4.6 shows the block diagram of the system built in SIMULAB. 


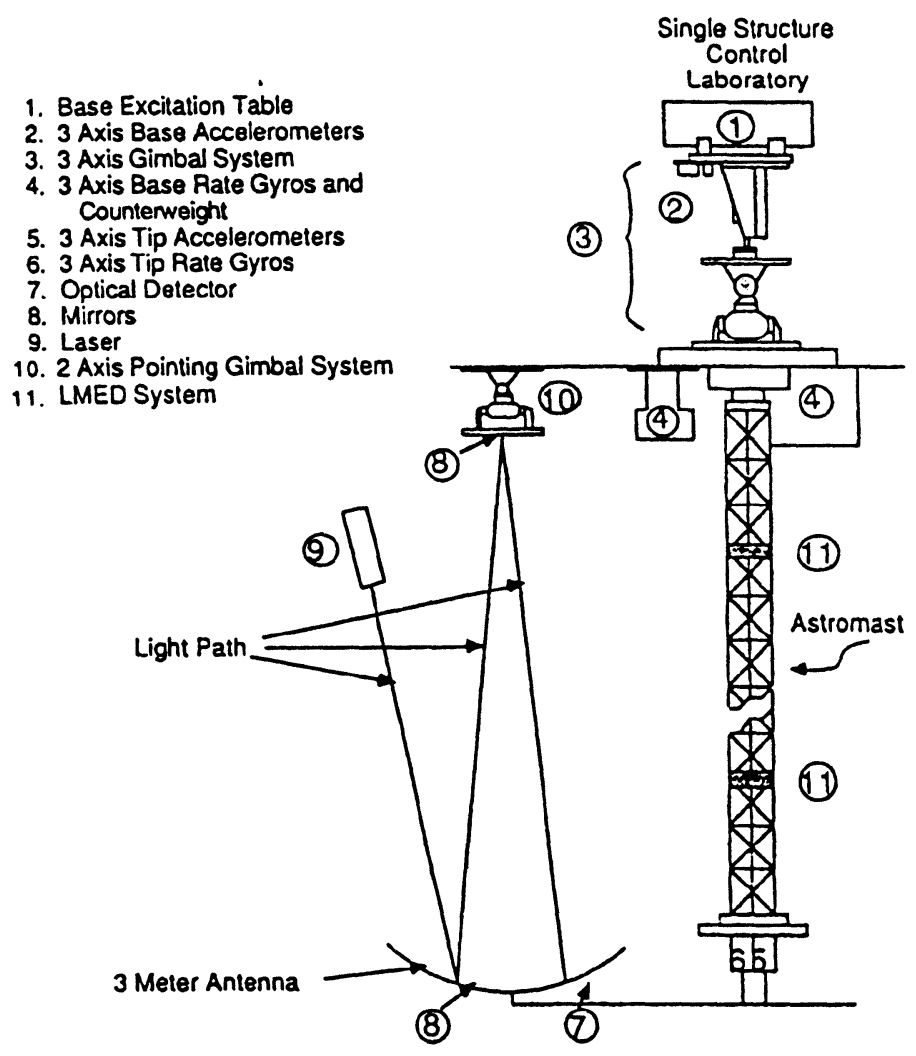

Figure 4.5: Marshall Space Flight Center Large Space Structure Ground Test Facility (ACES Configuration).

The SIMULAB toolbox is designed to interface directly with the Matlab workspace. The matrices representing the discrete realization, $\left(A_{d}, B_{d}, C_{d}, D_{d}\right)$, are calculated and stored in the Matlab workspace. These variables are then specified as the parameters of the Dis. State-space block in Figure 4.6. The white noise blocks are selected from the source component library of SIMULAB. The white noise sequences used in these blocks are the output of the Matlab "rand" function. The Constant Input block is also from the source component library. Any value can be specified in a constant block. For this simulation, zero was used since only one input should be excited at a time. The Sum, Mux, and Demux blocks are elements of the 


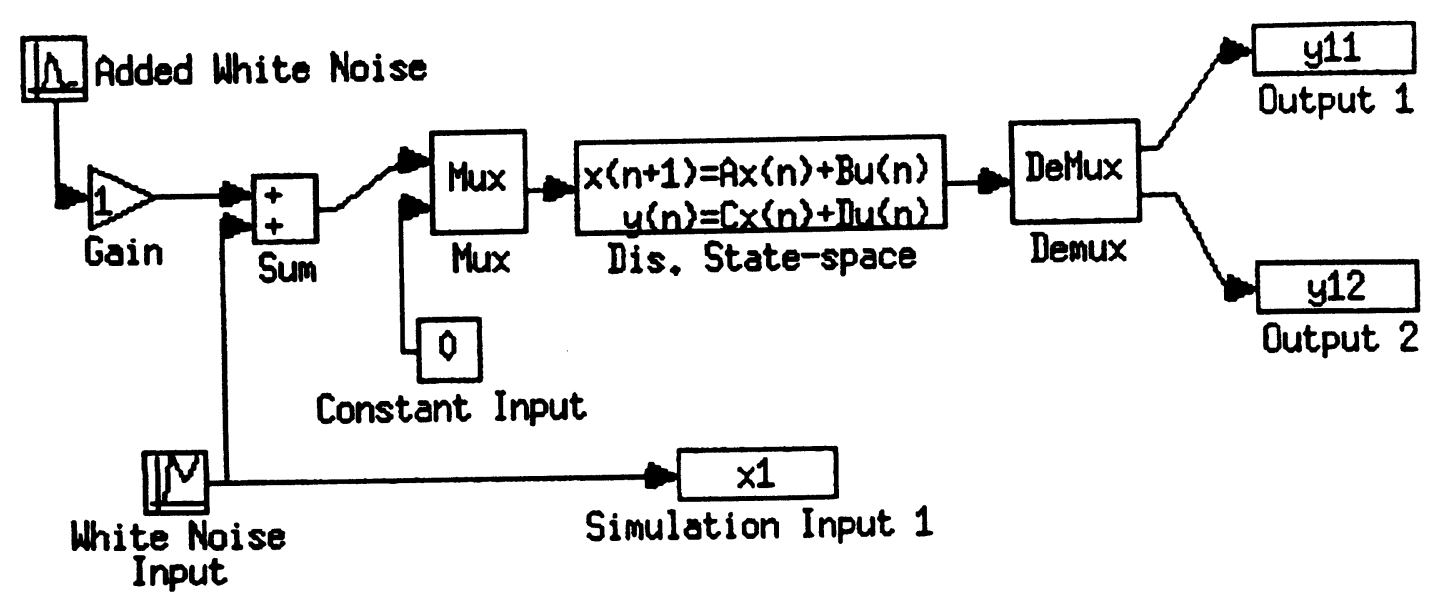

Figure 4.6: Block diagram of simulation using Matlab SIMULAB toolbox.

connections library. The Gain element was added to allow flexibility in varying the simulated noise level in the system. Finally three To Workspace blocks were selected from the sink component library, Simulation Input 1, Output 1, and Output 2, to collect the data from the simulation. These blocks store their data points back into the Matlab workspace with the variable names supplied in the block. When the simulation is started, SIMULAB obtains the required data from the Matlab workspace, performs the simulation, in this case a linear simulation, and then stores the output data back to the Matlab workspace. Therefore, after the simulation is complete, the variables $x 1, y 11$, and $y 12$ exist in the Matlab workspace and can be used as any other variable created by the user in Matlab. The simulation ran from zero to 100000 seconds. This allowed enough data so that various size FFT's could be calculated and results using different amounts of averaging could be compared. After performing the simulation on the system, an input and two output time records are available. At this point, the problem for TFDC is essentially the same as would be 
faced in an actual system; i.e., given input and output time domain data from the system, identify a transfer function which satisfactorily describes the characteristics of the system.

\subsection{Obtaining Frequency Response Data From Time Sequences}

Since TFDC is a frequency domain identification algorithm, frequency response data must be produced from the time data. The "spectrum" command from the Matlab Signal Processing Toolbox is used to produce the frequency response data. "Spectrum" performs power spectral analysis on two time sequences, $x$ and $y$, using the Welch method of power spectrum estimation. The two $n$ point sequences are divided into $m$ point sections. Successive sections are Hanning windowed, transformed to the frequency domain with an $m$-point FFT and accumulated. Confidence intervals are estimated by calculating the variance of the unaveraged spectral estimates under the assumption of a normal distribution. "Spectrum" returns an eight column matrix, where each row corresponds to individual frequency points and the elements of each column are described by the following:

Column $1=x$ power spectral density

Column $2=y$ power spectral density

Column $3=$ Cross spectral density

Column $4=$ Complex transfer function from $x$ to $y$

Column $5=$ Coherence function between $x$ and $y$

Column $6=95 \%$ confidence interval for $x$ power spectral density

Column $7=95 \%$ confidence interval for $y$ power spectral density

Column $8=95 \%$ confidence interval for cross spectral density

(Little, 1988) 
Two columns of data from the "spectrum" output matrix can be used as input to TFDC. These are column two, the power spectral density of the output sequence, and column four, the complex frequency response estimates from the input to the output of the system.

\subsection{Results from Applying TFDC to the Constructed Example System}

Using the method described in Section 4.1, time data for the example system was produced using the SIMULAB toolbox with Matlab. The noise sequence added to the input was Gaussian with zero mean, unity variance, and unity gain. Then, the experimental frequency response data was derived using the "spectrum" command from Matlab as shown in Section 4.3. This produced the complex frequency response data and the output power spectral density data vectors which can be used as input to TFDC. First, TFDC was applied to the complex frequency response data using the TLS and LS/TLS solutions and these results were compared to the LS solution.
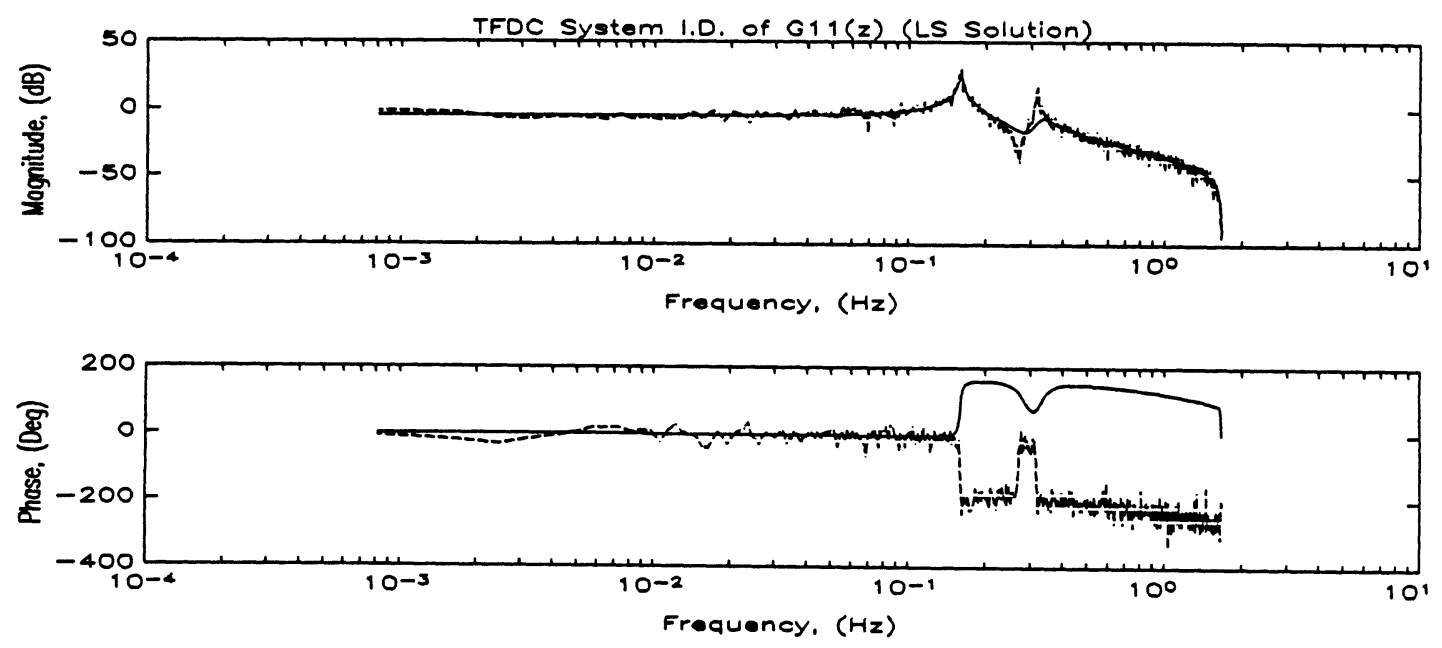

Figure 4.7: Complex Transfer Function System I.D. (LS Solution). 

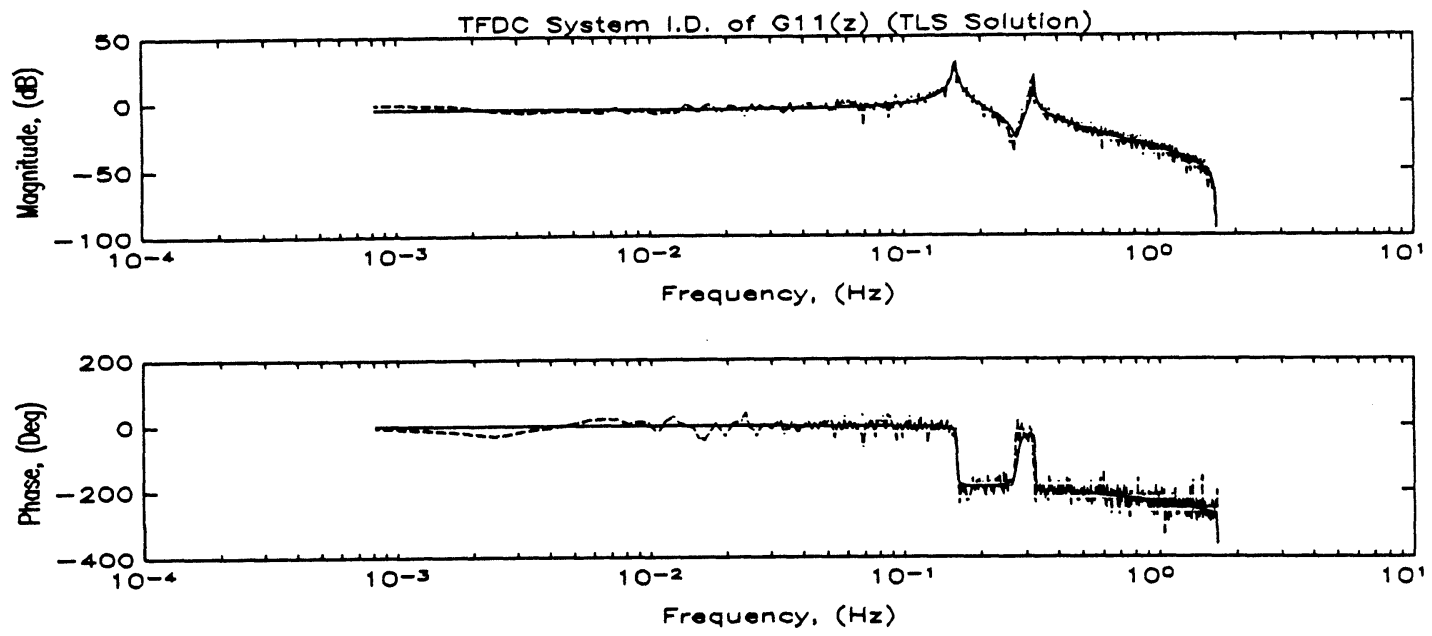

Figure 4.8: Complex Transfer Function System I.D. (TLS Solution).
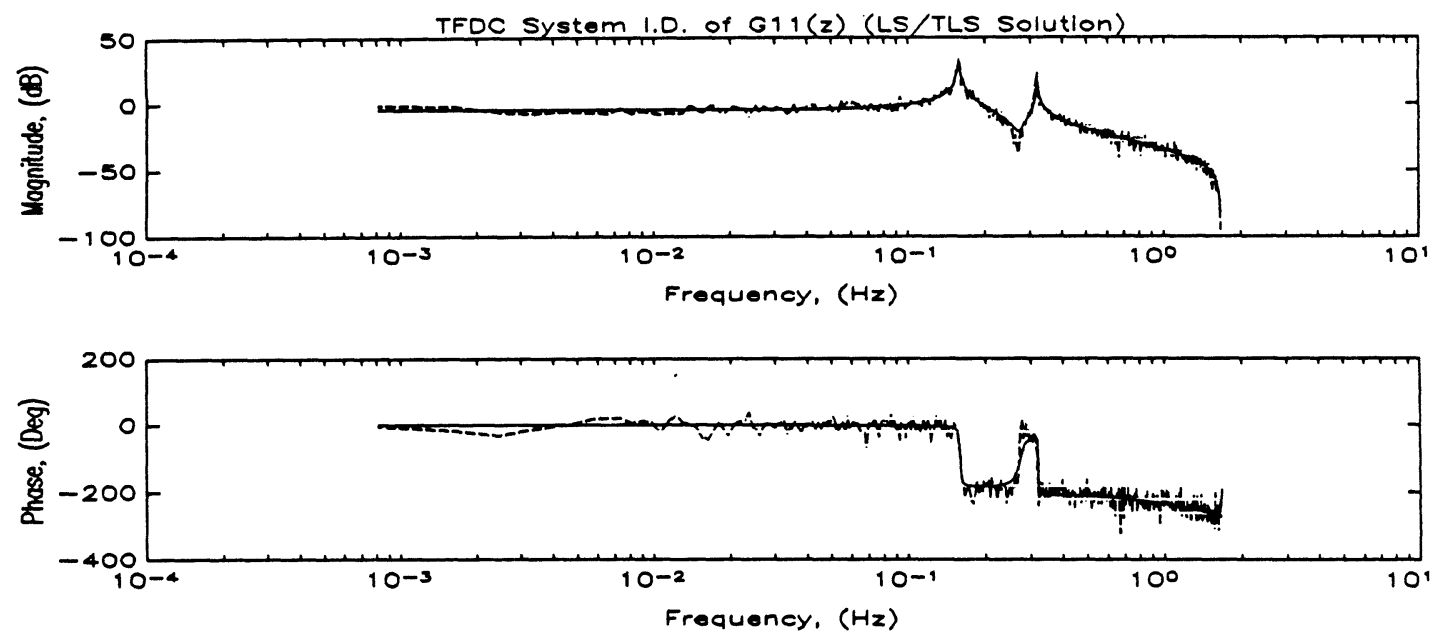

Figure 4.9: Complex Transfer Function System I.D. (LS/TLS Solution).

Figure 4.7 - Figure 4.9 compare the results of applying TFDC $\left(4^{\text {th }}\right.$ order model assumed) to the complex frequency response data, $G_{I I}\left(e^{j \omega T}\right)$, obtained via "spectrum". Figure 4.7 compares the experimental frequency response with the frequency response of the identified transfer function using an LS solution. 
Figure 4.8 and Figure 4.9 compare the same frequency responses when TLS and LS/TLS solutions are used in TFDC. As seen in Figure 4.7, the LS solution does not provide an acceptable transfer function to describe the system. The system mode and zero near $.3 \mathrm{~Hz}$. are more heavily damped in the identified frequency response than in the actual data and the phase is not even close to describing the actual data. In Figure 4.8, though, the TLS solution provides an acceptable model for the system. Both of the system modes are identified and the zero, while still more heavily damped than in the actual data, is close to the actual data. The phase in the TLS solution also closely follows the actual data. It is also interesting to note that the model from the TLS solution contains all stable system poles while the LS solution produced a model in which all the system poles were outside the unit circle.

The LS/TLS solution, Figure 4.9, produced an identified transfer function which is almost identical to the transfer function identified with the TLS solution shown in Figure 4.8. In fact, although not proved analytically, the LS/TLS solution always produced transfer functions almost identical to the those of the TLS solution as shown in Table 4.1. In order to compare the TLS and LS/TLS solutions, the system identification process was performed seven times each with the TLS and LS/TLS solution methods while varying the amount of averaging. The amount of averaging was varied by taking FFT's of varying length, thus varying the number of sequences averaged together, in the "spectrum" command. All other parameters in the identification process were held constant. The 2-norm was used in determining that the solutions were the same. The data in column three of Table 4.1 were calculated by 


\begin{tabular}{||c|c|c|c||}
\hline $\begin{array}{c}\text { Number of } \\
\text { Averages }\end{array}$ & $\begin{array}{c}\text { Number of FFT } \\
\text { points used }\end{array}$ & $\begin{array}{c}\text { 2-Norm of Numerator } \\
\text { Coefficient Error }\end{array}$ & $\begin{array}{c}\text { 2-Norm of Deno- } \\
\text { minator Coefficient } \\
\text { Error }\end{array}$ \\
\hline 196 & 512 & $2.2203 \mathrm{e}-6$ & $1.5487 \mathrm{e}-5$ \\
\hline 98 & 1024 & $2.2906 \mathrm{e}-6$ & $1.2010 \mathrm{e}-5$ \\
\hline 49 & 2048 & $2.3585 \mathrm{e}-6$ & $1.3121 \mathrm{e}-5$ \\
\hline 25 & 4096 & $2.2771 \mathrm{e}-7$ & $7.4967 \mathrm{e}-7$ \\
\hline 13 & 8192 & $3.4504 \mathrm{e}-6$ & $1.2400 \mathrm{e}-5$ \\
\hline 7 & 16384 & $4.6269 \mathrm{e}-6$ & $1.1897 \mathrm{e}-5$ \\
\hline 4 & 32768 & $7.6357 \mathrm{e}-6$ & $1.3113 \mathrm{e}-5$ \\
\hline
\end{tabular}

Table 4.1: Comparison of coefficients determined from TLS and LS/TLS solutions.

taking the 2-norm of the difference of the numerator coefficient vectors from each solution method; i.e., $\|t l s-l s t l s\|_{2}$. Column four was calculated in the same manner for the denominator coefficients.

Figure 4.10 - Figure 4.12 compare the results of applying TFDC, using the LS, TLS, and CLS solutions, respectively, to the power spectral density data of the example system output one when the system is excited at input one. The LS/TLS solution is not presented since it produces the same results as the TLS solution. The three solutions yield transfer functions with very similar magnitude and phase characteristics. The TLS and CLS solutions in Figure 4.11 and Figure 4.12 have nearly identical magnitude and phase functions. Similar to the transfer function data in Figure 4.7, the zero near .3 Hz. in the LS solution in Figure 4.10 is more heavily damped than the actual data shows. In the case of psd data, though, neither the TLS 

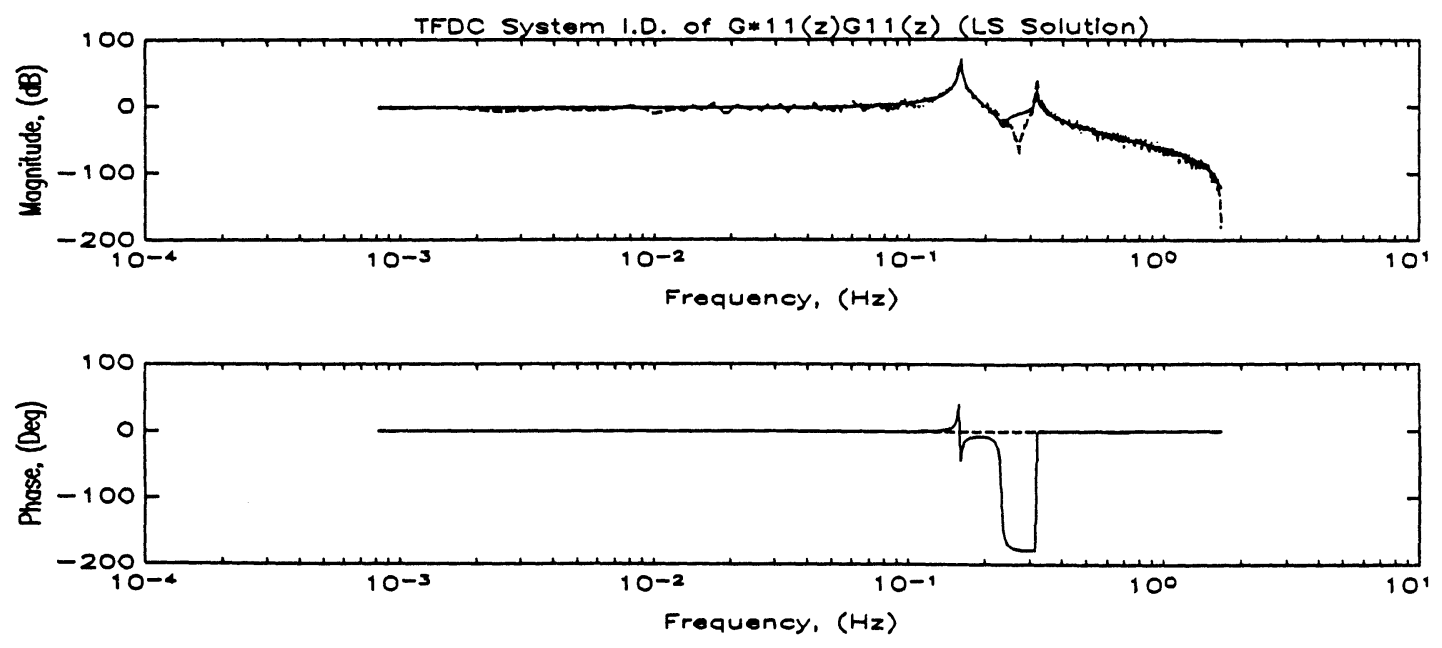

Figure 4.10: Power Spectral Density System I.D. (LS Solution).
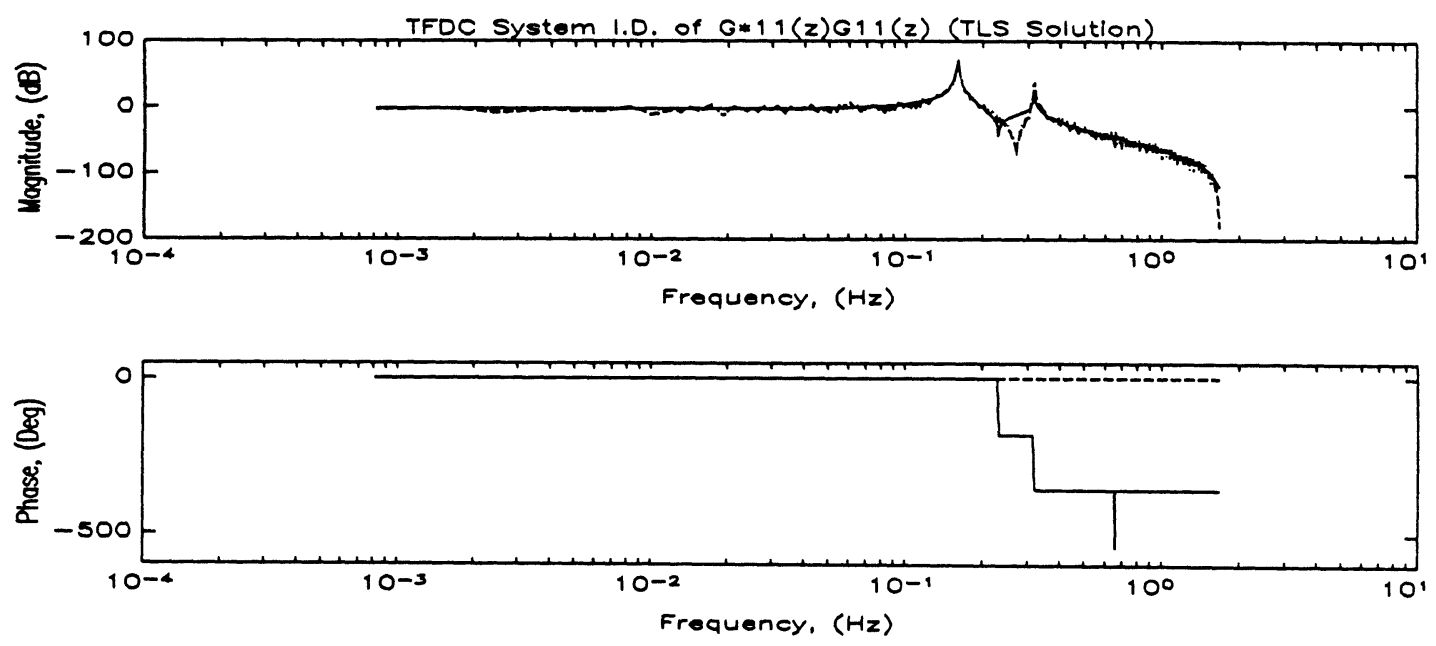

Figure 4.11: Power Spectral Density System I.D. (TLS Solution).

nor the CLS solutions produced much better results. The LS solution produced six unstable poles, the TLS solution produced two unstable poles, and the CLS solution produced four unstable poles in the z-plane. The CLS solution provided the best model of the three since it is expected to have four poles inside the unit circle and 

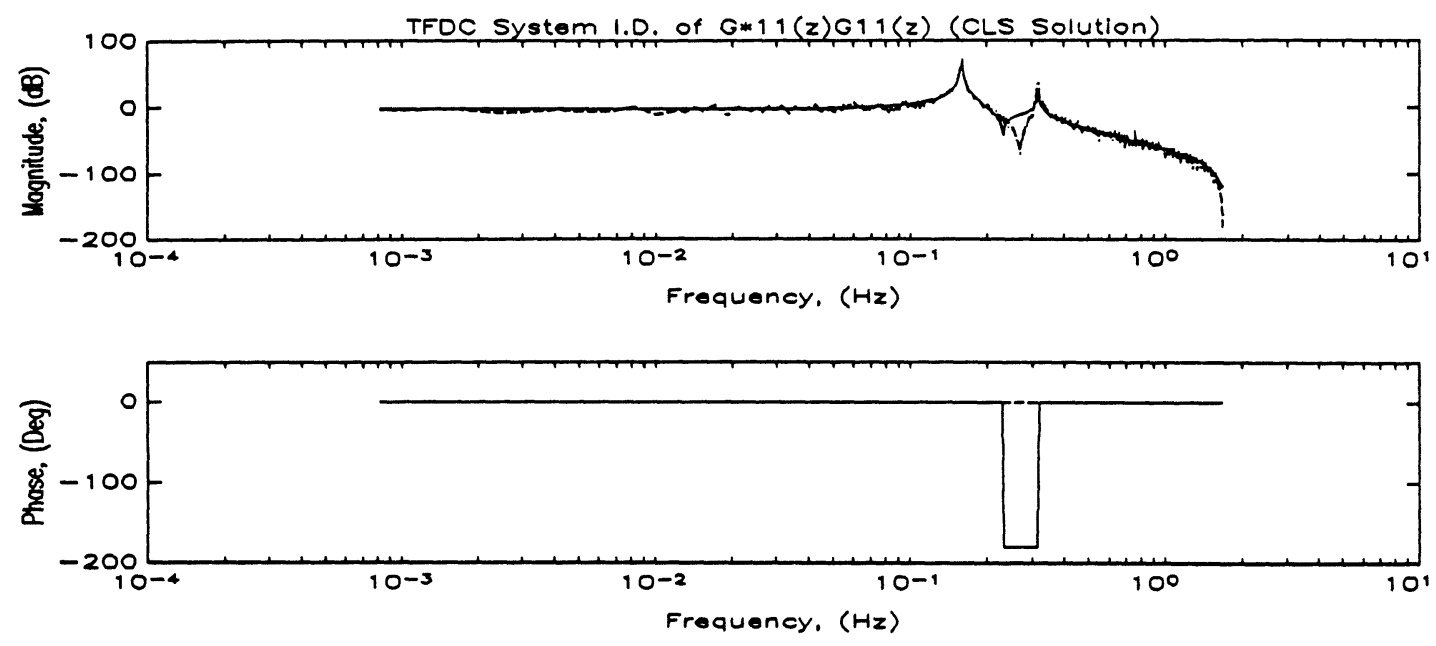

Figure 4.12: Power Spectral Density System I.D. (CLS Solution).

four poles outside the unit circle when dealing with psd data. The LS solution is not usable because a system transfer function derived from this solution would contain at least two unstable poles. Since the TLS solution only produces two unstable poles, the poles of the psd rational function will not have the expected quadrantal symmetry in the w-plane. Therefore the CLS solution would be chosen as the best model of the psd data.

\subsection{Results from Applying TFDC to the AGS Subsystem Data}

Time domain data was supplied from NASA for the AGS subsystem of the ACES facility. This data was processed using "spectrum" from Matlab as described in Section 4.3 to produce the complex frequency response data needed for input to TFDC. Figure 4.13 and Figure 4.14 show the results of applying TFDC (LS and TLS solutions, respectively) to the data from the AGS $\mathrm{x}$ axis actuator to the BG $\mathrm{x}$ axis sensor. Figure 4.15 and Figure 4.16 show the same results for the y axis actua- 
tor/sensor pair. TFDC attempted to identify a 58th order system from the AGS/BG subsystem data. The LS solution for the $\mathrm{x}$ axis identified a system with 48 unstable poles and for the y axis the identified transfer function had 46 poles outside the unit circle. Although the TLS solutions for the $\mathrm{x}$ and $\mathrm{y}$ axes produced unstable transfer functions for both, there were only 8 unstable poles for the $\mathrm{x}$ axis and 9 unstable poles for the y axis.
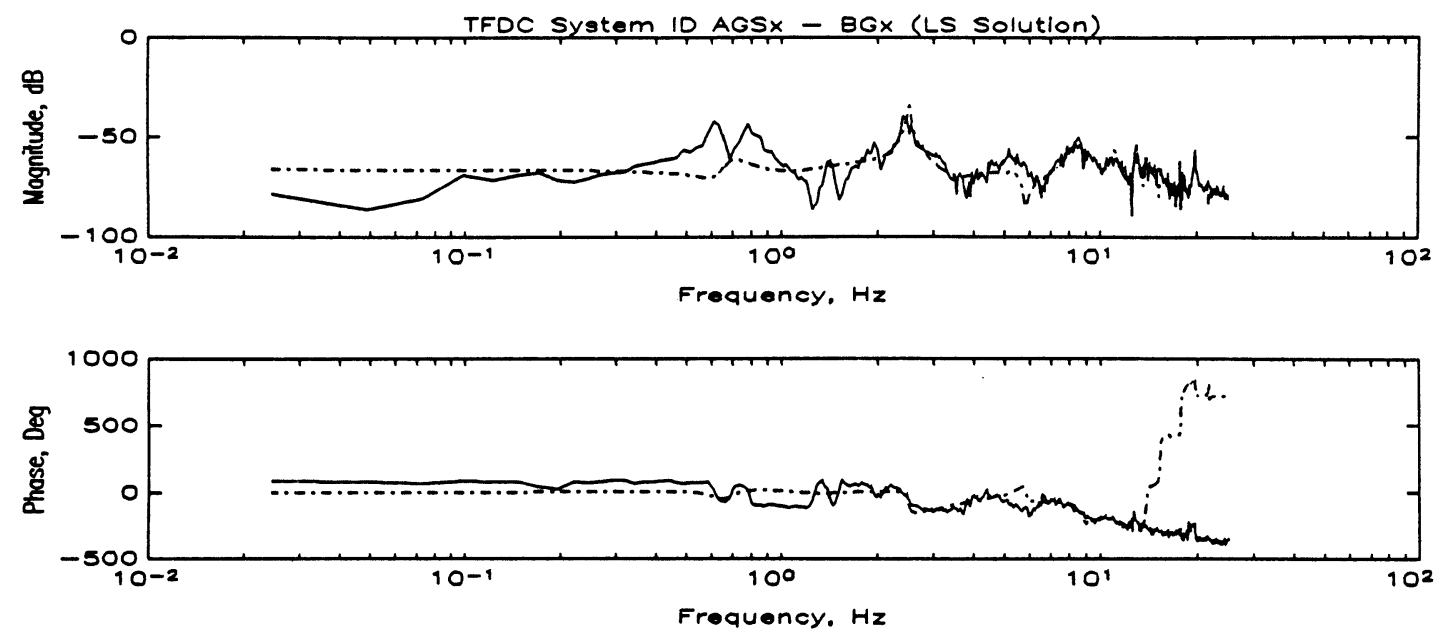

Figure 4.13: Complex Transfer Function System I.D. AGSx to BGx. (LS Solution)

TFDC, using the TLS solution applied to the AGS/BG subsystem data, produced a much more accurate transfer function than TFDC with the LS solution. Both solutions were fairly accurate at high frequency. The decades of lower frequency are where the identified transfer functions were inaccurate at describing the actual system data. As seen in the plots, at low frequency, the TLS solutions, while still inaccurate, produced a much better description of the actual data than did the LS solutions. This inaccuracy is inherent in all the least squares solution methods 

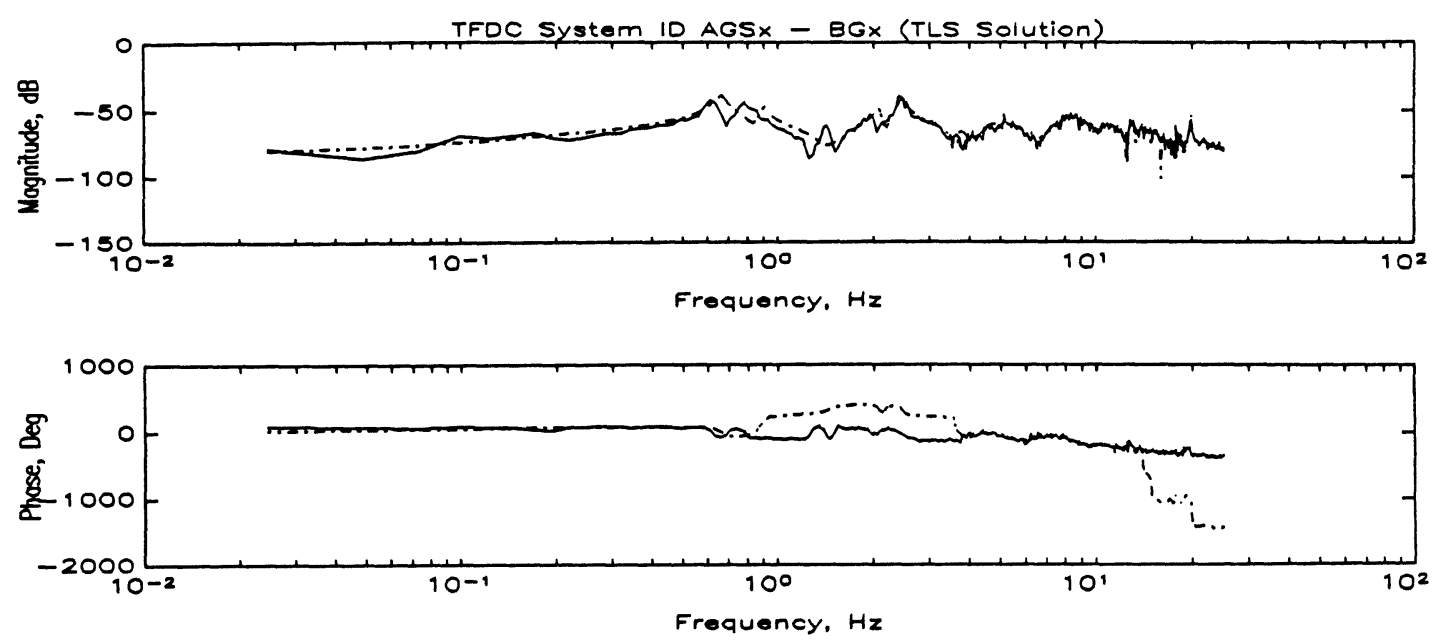

Figure 4.14: Complex Transfer Function System I.D. AGSx to BGx (TLS Solution).
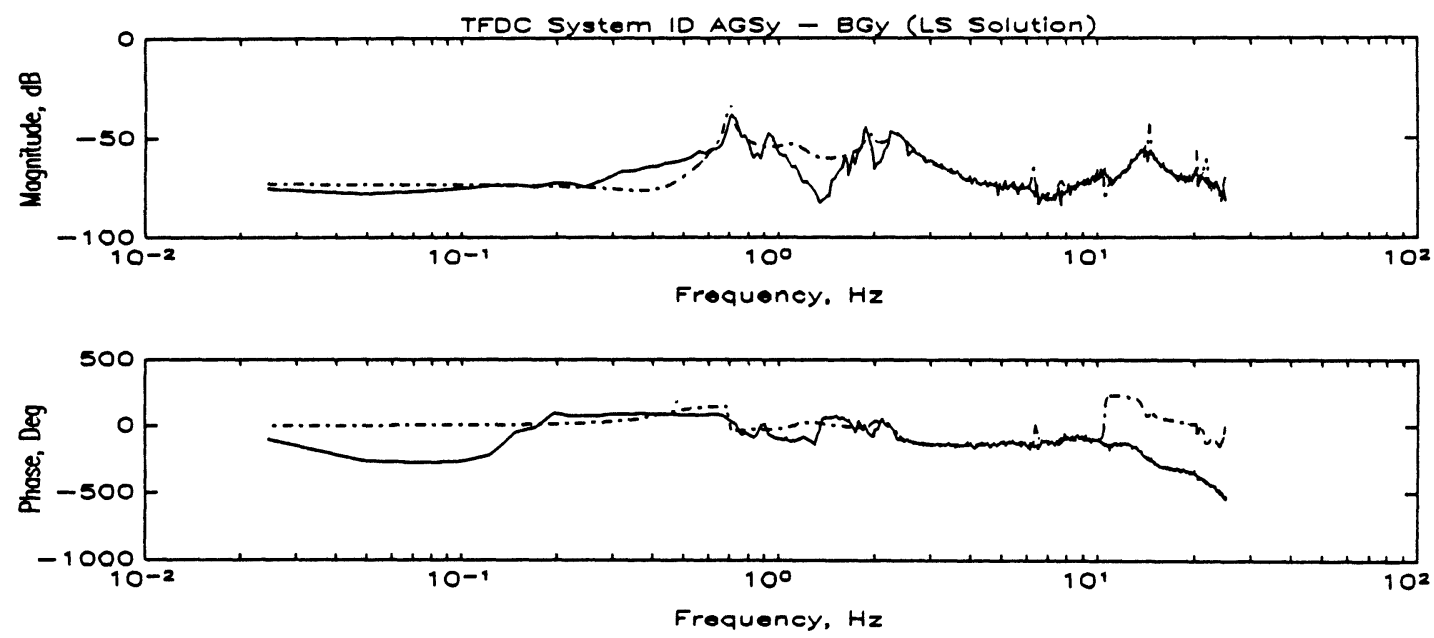

Figure 4.15: Complex Transfer Function System I.D. AGSy to BGy (LS Solution).

implemented. This seems to be due to the smaller number of data points in the low frequency decades. Thus the least squares solutions seem to emphasize the frequency decades which contain a larger number of points. This problem might be corrected by adding more points to the low frequency decades via interpolation between the actual points. 

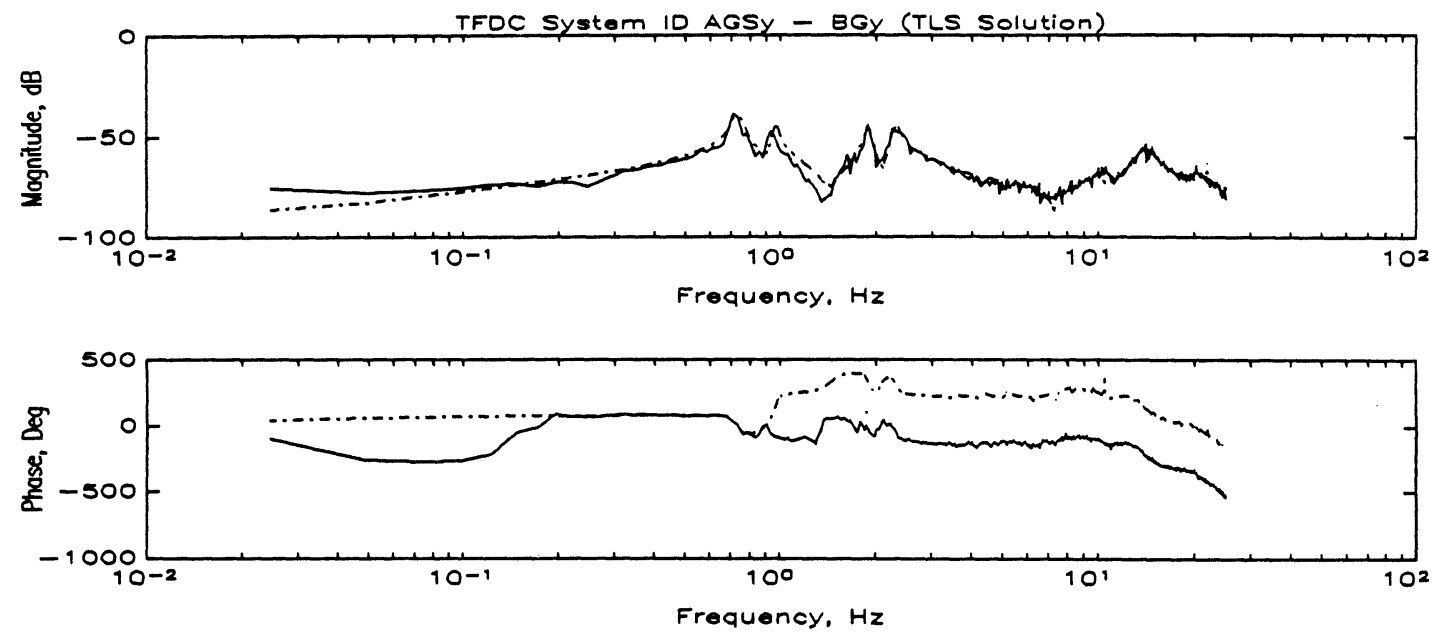

Figure 4.16: Complex Transfer Function System I.D. AGSy to BGy (TLS Solution).

In conclusion, the AGS/BG transfer functions identified using the TLS solution in TFDC are not acceptable since they contain unstable poles. These solutions, though, are, by far, better than the LS solutions used in the past. 


\section{Chapter 5: Conclusions and Recommendations}

The Transfer Function Determination Code (TFDC) is a single-input, singleoutput system identification algorithm. TFDC identifies input, output relationships in the form of a system transfer function from experimental frequency response data. The purpose of this thesis is to improve the accuracy of the identified transfer function by replacing the ordinary Least Squares (LS) method currently used with other least squares methods. The heart of the TFDC algorithm lies in the solution to an overdetermined system of linear equations, $A x \approx b$. Since the elements of the coefficient matrix and the right hand side vector are derived from the experimental frequency response data, these elements contain uncertainty due to noise in this data. In this case, the TLS solution should provide a more accurate solution since the TLS method accounts for uncertainty in the coefficient matrix as well as the right hand side vector. In addition to TLS, two other least squares methods, the combined Least Squares/Total Least Squares (LS/TLS) solution and the Constrained Least Squares (CLS) solution, were implemented in an attempt to improve the accuracy of the identified transfer function. Also, other code modifications were made to increase speed and efficiency of the TFDC algorithm.

First, the theory for TLS, LS/TLS, and CLS methods was presented in Chapter 2. Then, in Chapter 3, the TFDC algorithm was discussed along with a description of the modifications made in the code. As mentioned above, the heart of the TFDC algorithm is the solution to the system of linear equations. These equations are derived in Section 3.1. A brief history is given explaining the application and 
problems of the LS solution previously used. Finally, modifications made to decrease the code execution time are explained along with the algorithms used in finding the TLS, LS/TLS, and CLS solutions.

To test the modified code and verify the improved accuracy of the TLS solution, TFDC was applied to a constructed example system. This two input, two output system was simulated and an experimental frequency response obtained. TFDC, using the TLS solution, produced a stable system transfer function whose frequency response closely matched the true frequency response of the system. For the same system, the LS solution produced a less accurate and unstable model of the system. Also the new version of TFDC executes more quickly and require much less user interaction since no weighting or iteration is used.

TFDC can also be applied to power spectral density data from the output sequence. When applied to output psd data assuming the input was a white noise sequence, TFDC attempts to identify the rational function $G\left(e^{-j \omega T}\right) G\left(e^{j \omega T}\right)$. This function is expected to have quadrantal symmetry of its poles and zeros. The CLS solution method was applied so that the poles and zeros of this function are constrained to have quadrantal symmetry. When applied to the constructed example, it was found that TFDC does produce a rational function with the required symmetry, but the frequency response of this function did not acceptably match the experimental psd data. This solution method, though, was the best among the LS, TLS, and CLS solutions methods. This method could be improved upon by applying weighting and 
iteration just as the LS solution has been improved in the past with weighting and iteration.

Finally the TFDC code was applied to experimental data supplied by NASA. TFDC with TLS did not identify a completely acceptable transfer function to describe the system, but the TLS solution worked much better the LS solution which has been tried in the past.

The work presented in this thesis shows positive results when applying a TLS solution to the system of equations in the TFDC algorithm. For the systems investigated, the TLS solution produced a more accurate transfer function than the LS solution. In addition, the TLS method required much less user interaction and decision making and executes much faster than the LS solution. The LS/TLS solution method was implemented, but, for the constructed example system, this solution provided no benefit since it was essentially identical to the TLS solution. The CLS solution did not identify acceptable transfer functions for psd data, but if weighting and iteration are applied, it is likely that more accuracy could be achieved. Future work on TFDC could include the following:

1. Implementation of a weighted CLS solution method. This should improve the accuracy of the CLS solution while still insuring the pole and zero symmetry required when applying TFDC to output psd data.

2. Further decreases in the execution time might be achieved by using mex files in Matlab and converting the code to Matlab version 4. Mex files are compiled $\mathrm{C}$ or Fortran subroutines which can be executed without the overhead 
of the Matlab command line parser. Matlab version 4 makes use of sparse matrix storage and computations. This would allow larger amounts of data to be used and would allow the use of the fastest method in building the linear system.

3. Finding a better frequency selection algorithm.

4. Addition of frequency interpolation in frequency decades where there are few data points. 


\section{References}

Eckart, G. and Young, G. (1936), "The approximation of one matrix by another of lower rank", Psychometrica, 1, pp. 211-218.

Golub, G. H., Hoffman, A., and Stewart, G. W. (1987), "A generalization of the Eckart-Young-Mirsky matrix approximation theorem", Linear Algebra Applications 88/89.

Golub, G. H. and Van Loan, C. F. (1989), Matrix Computations, Second Edition, The Johns Hopkins University Press, Baltimore, MD.

The Mathworks, Inc. (1990), PRO-MATLAB User's Guide, South Natick, MA.

The Mathworks, Inc. (1990), SIMULAB User's Guide, Natick, MA.

Lam, Harry Y-F. (1979), Analog and Digital Filters Design and Realization, Prentice-Hall, Inc., Englewood Cliffs, N.J.

Little, J and Shure, L. (1988), Signal Processing Toolbox for use with Matlab, The Mathworks, Inc., South Natick, MA.

Medina B., E. A. (1991), "Multi-input, Multi-output System Identification for Frequency Response Samples with Applications to the Modeling of Large Space Structures", Master of Science Thesis, Ohio University, November, 1991.

Mirsky, L. (1960), "Symmetric gauge functions and unitarily invariant norms", Quart. J. Math Oxford, 11, pp. 50-59.

Thompson, R. C. (1972), "Principal submatrices IX: interlacing inequalities for singular values of submatrices", Linear Algebra Applications, 5, pp. 1-12.

Van Huffel, S. and Vandewalle, J. (1989), "Analysis and properties of the generalized total least squares problem $A X \approx B$ when some or all columns of $A$ are subject to errors", SIAM J. Marrix Anal. Appl., 10, pp. 294-315.

Van Huffel, S. and Vandewalle J. (1991), The Total Least Squares Problem:

Computational Aspects and Analysis, Society for Industrial and Applied Mathematics, Philadelphia, PA. 


\section{Appendix}

The Transfer Function Determination Code (TFDC) has been implemented via a set of Matlab $\mathrm{m}$-files. These consist of a main and 4 subfunctions. The main module is contained in the m-file "tfdc.m". The subfunction "tlssolvr" calculates the TLS solution to an overdetermined system of linear equations. The subfunctions "buildco", "coeff", and "binom" work together to construct the constraint equations which insure quadrantal pole, zero symmetry in the CLS solution. Section A.1 contains the source code for the m-file containing the main code module "tfdc." Sections A.2 - A.5 contain, respectively, the "tlssolvr", "buildco", "coeff", and "binom" subfunctions. 


\section{A.1. "TFDC.M"}

$\%$ M-File to identify siso transfer functions from data contained

$\%$ in columns 4 and 5 of the "p arrays" generated by SPECTRUM.

$\%$ Use as [num,den] $=\operatorname{tfdc}(p$, norder, $w, f s$, npts, method,tfname $)$

$\% \mathrm{p}$ is a matrix whose first column contains the frequency response

$\%$ in complex form and whose second column contains the coherence function

$\%$ norder is the order of the model (must be even order)

$\% \mathrm{w}$ is the frequency vector $(\mathrm{rad} / \mathrm{sec})$

$\%$ fs is the sampling frequency $(\mathrm{Hz})$

$\%$ npts is the number of linear and logarithmically space points to be used $\%$ method is the solution method to be used in solving the equations and can $\%$ be " $t$ ", "l", "c", or "s" for tls, lstls, cls, or $1 s$, respectively $\%$ tfname is a title for output plots (use', to input)

function $[$ num,den $]=\operatorname{tfdc}(p$, norder, $w, f s$, numpts, method, tfname $)$

$\mathrm{ii}=$ input('Do you want to load the variables of a previous run? $y / n[n]: ', ' s ')$;

if ii $==$ 'y'; \%***** the initial variables are loaded

clc

disp('Load your variables now, and we will go to the modeling part !') keyboard

else $\% * * * * *$ the initial variables are created

num $=$ length $(\mathrm{p}(:, 1))$;

deltaf $=\mathrm{fs} /(4 *$ num $)$;

$\mathrm{w}=\mathrm{w}(2: \mathrm{num}) /(2 * \mathrm{pi})$;

$\% \mathrm{w}=($ linspace $(\mathrm{fs} /(2 *$ num $), \mathrm{fs} / 2$, num- 1$))$;

$\operatorname{magdb}=20 .{ }^{*} \log 10(\operatorname{abs}(\mathrm{p}(2: \mathrm{num}, 1)))$;

phasedeg $=(180 . / \mathrm{pi}) *$ angle $(\mathrm{p}(2:$ num, 1$))$;

$\%$ coherence $=\mathrm{p}(2:$ num, 2$)$;

clg

hold off

$\%$

$\%$ display experimental frequency response data

$\%$

subplot(211), semilogx(w,magdb,' +');

xlabel('FREQUENCY (HZ)')

ylabel('MAGNITUDE (DB)')

text(.25,.46, ${ }^{* * *}$ USE MOUSE TO CHOOSE FREQUENCY RANGE***','sc')

subplot(212), semilogx(w,phasedeg,' +');

xlabel('FREQUENCY (HZ)') 


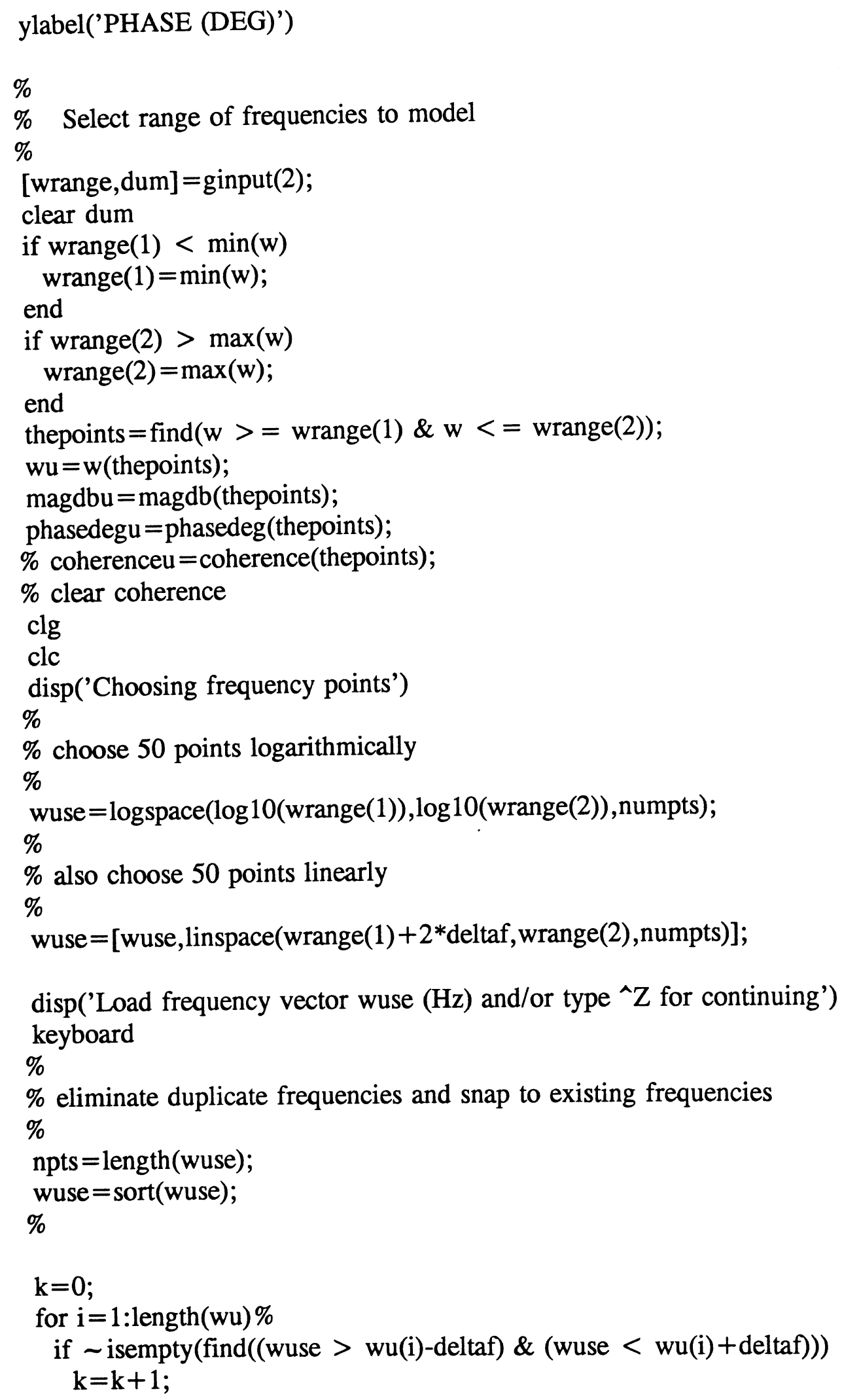




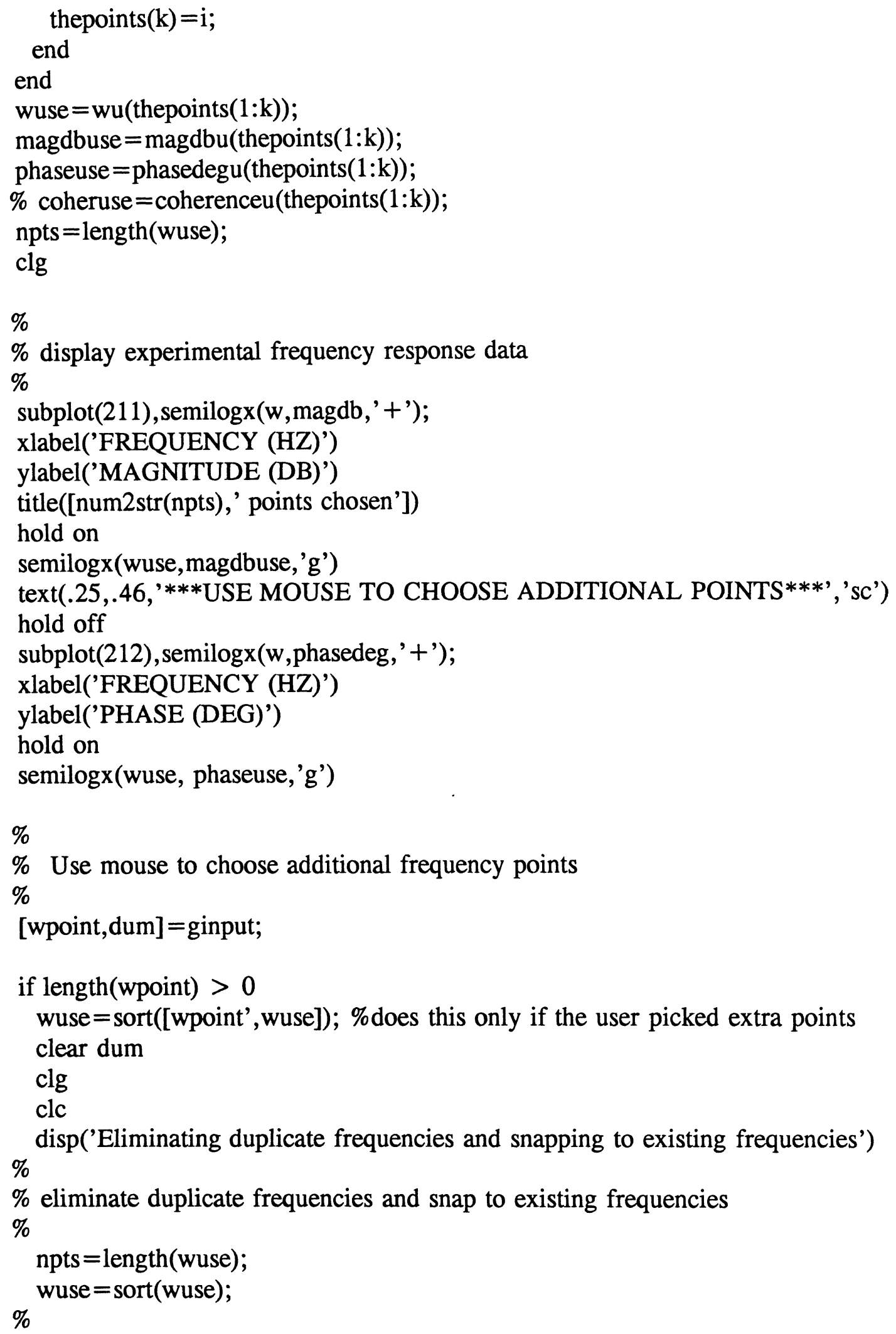




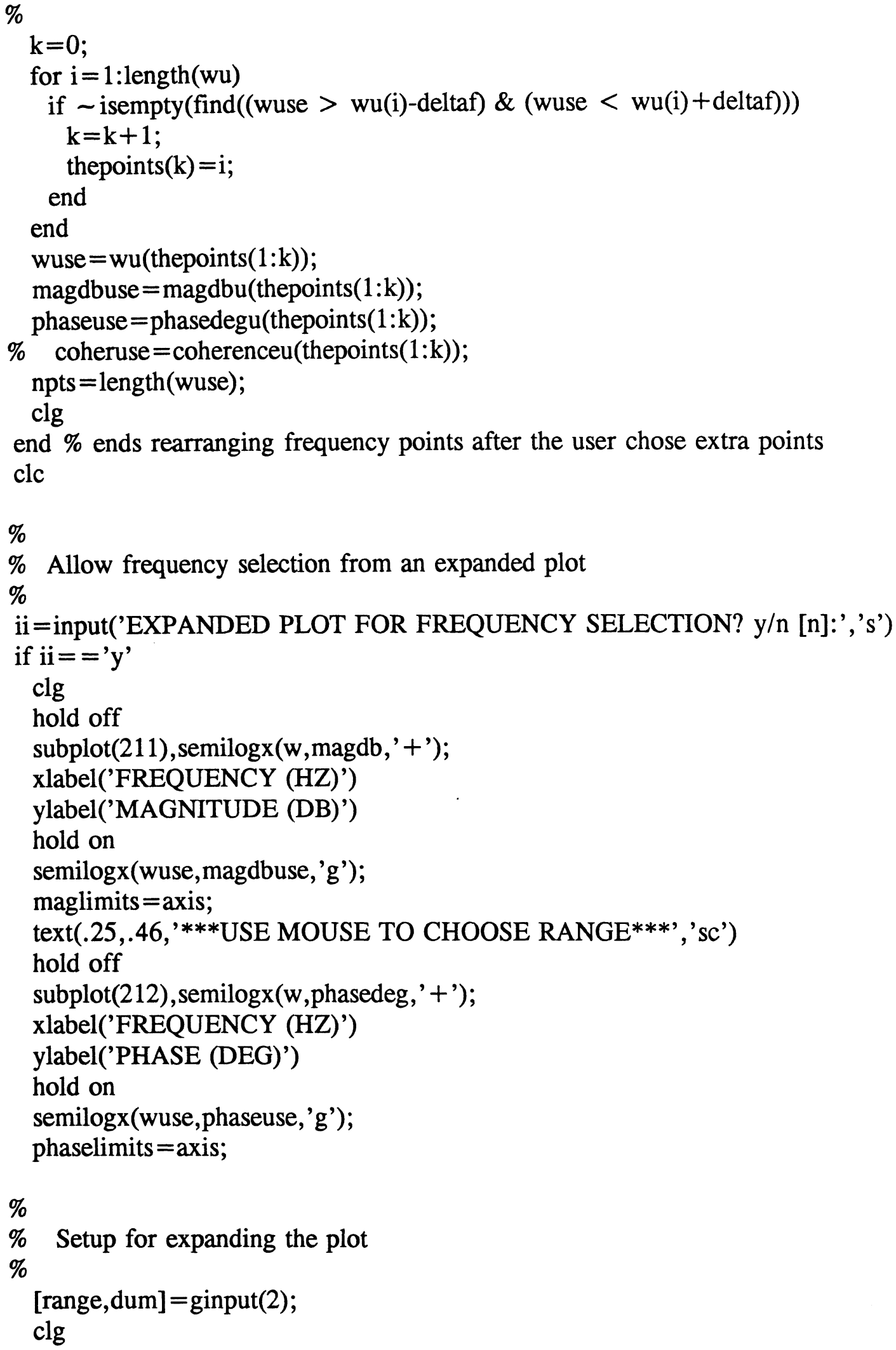




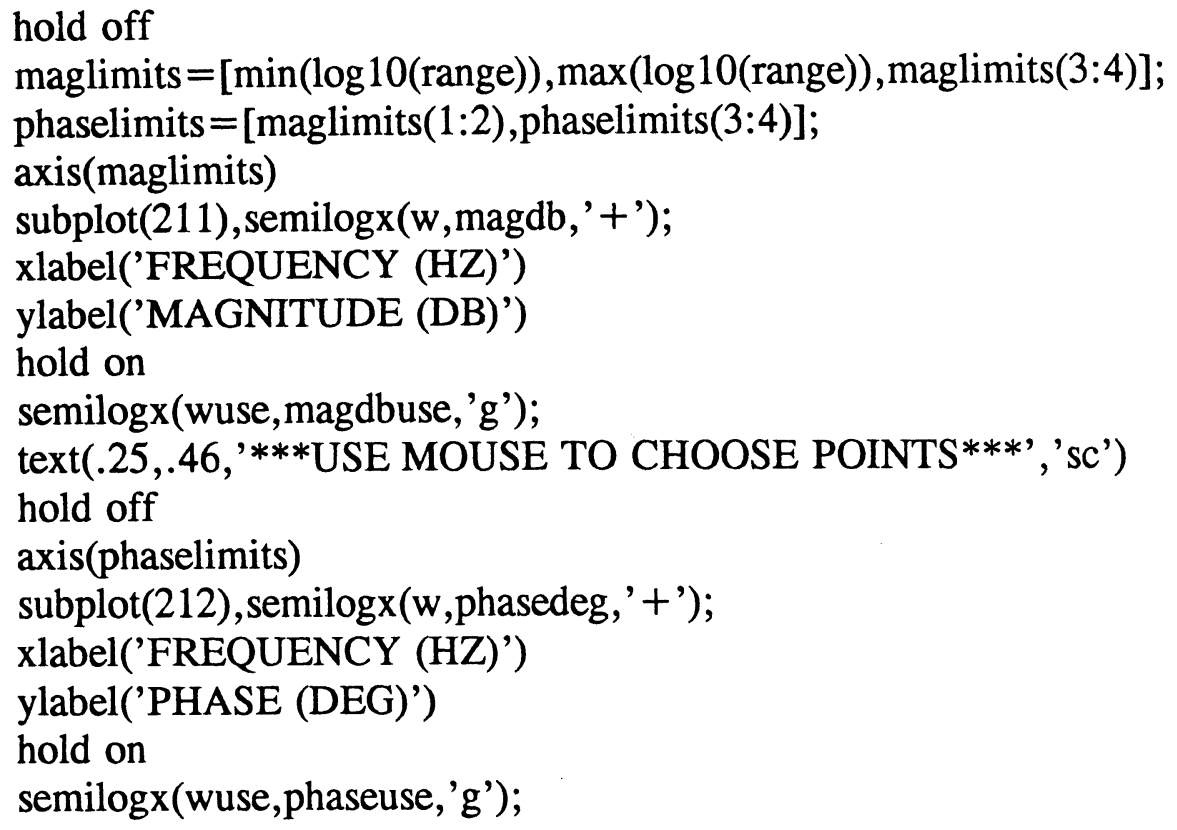




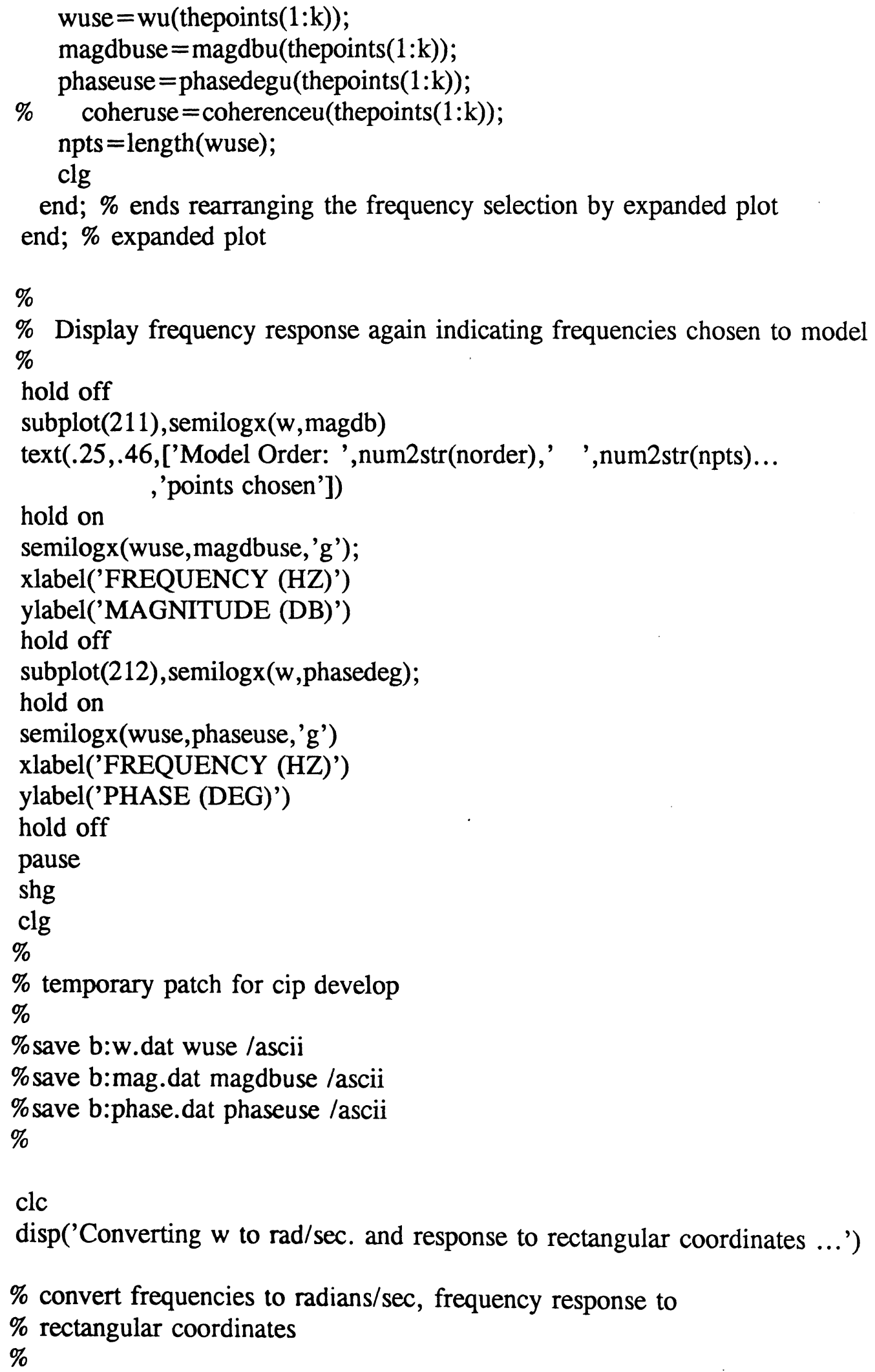




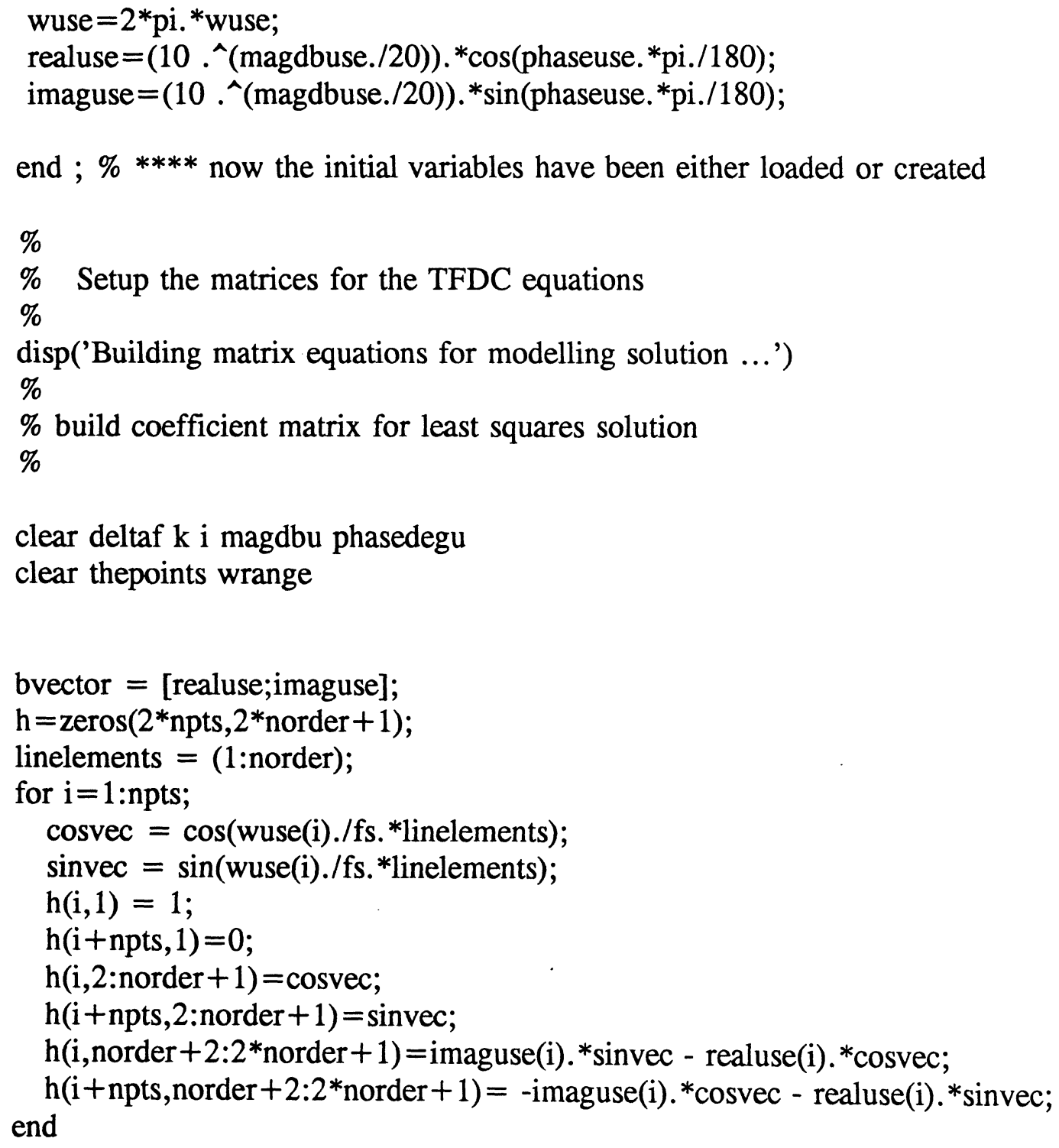

clear t1 t0 sinvec cosvec

$\%$

$\%$ Solve the system of equations using the method indicated on the command line $\%$

if method $==$ ' $t$ ', disp('Calculating TLS solution')

$\mathrm{x}=$ tlssolvr(h,bvector);

disp('TLS solution finished, extracting coefficients and displaying plots') 

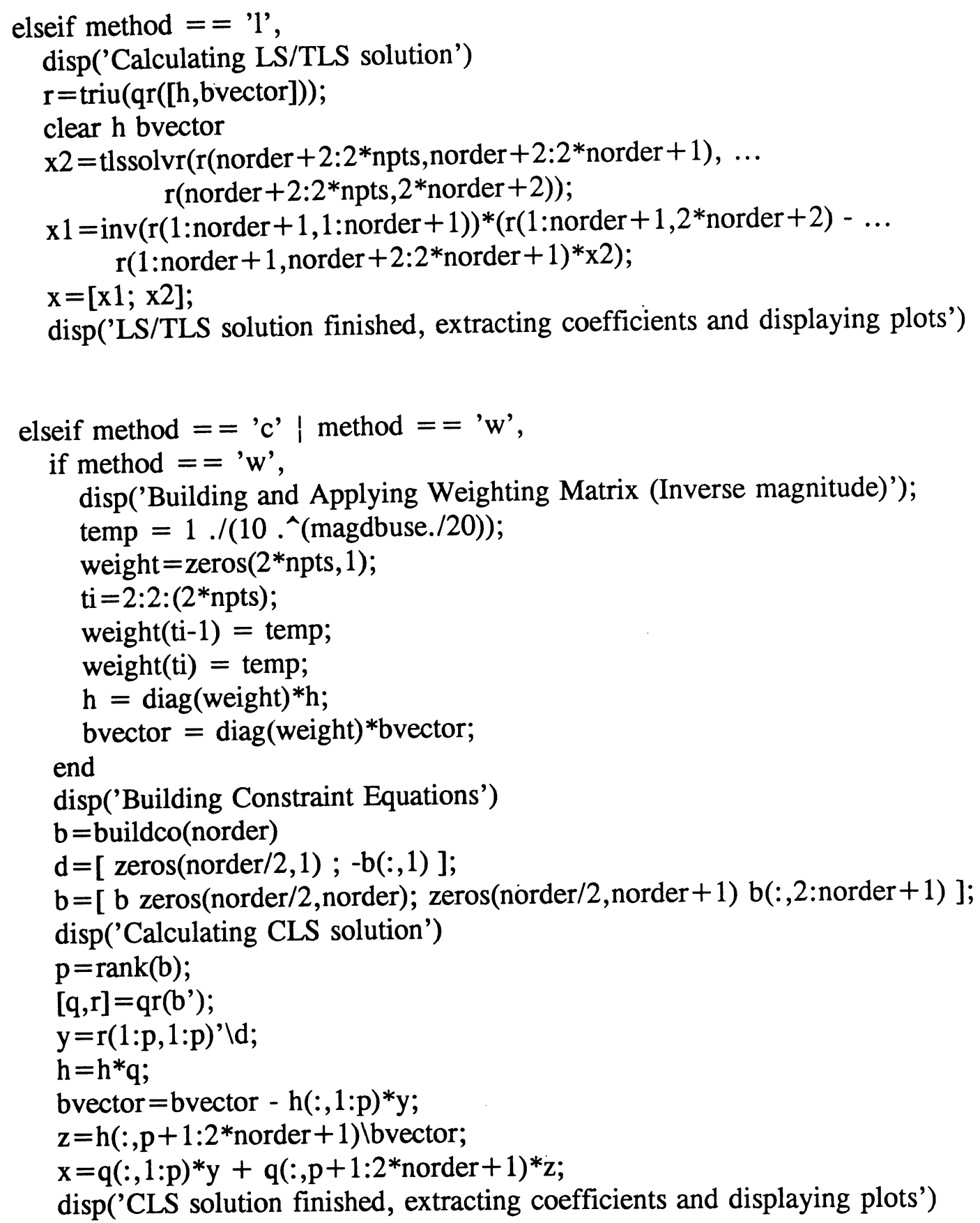

elseif method $==$ 's', disp('Calculating LS solution')

$\mathrm{x}=\mathrm{h} \backslash \mathrm{bvector}$ disp('LS solution finished, extracting coefficients and displaying plots') 


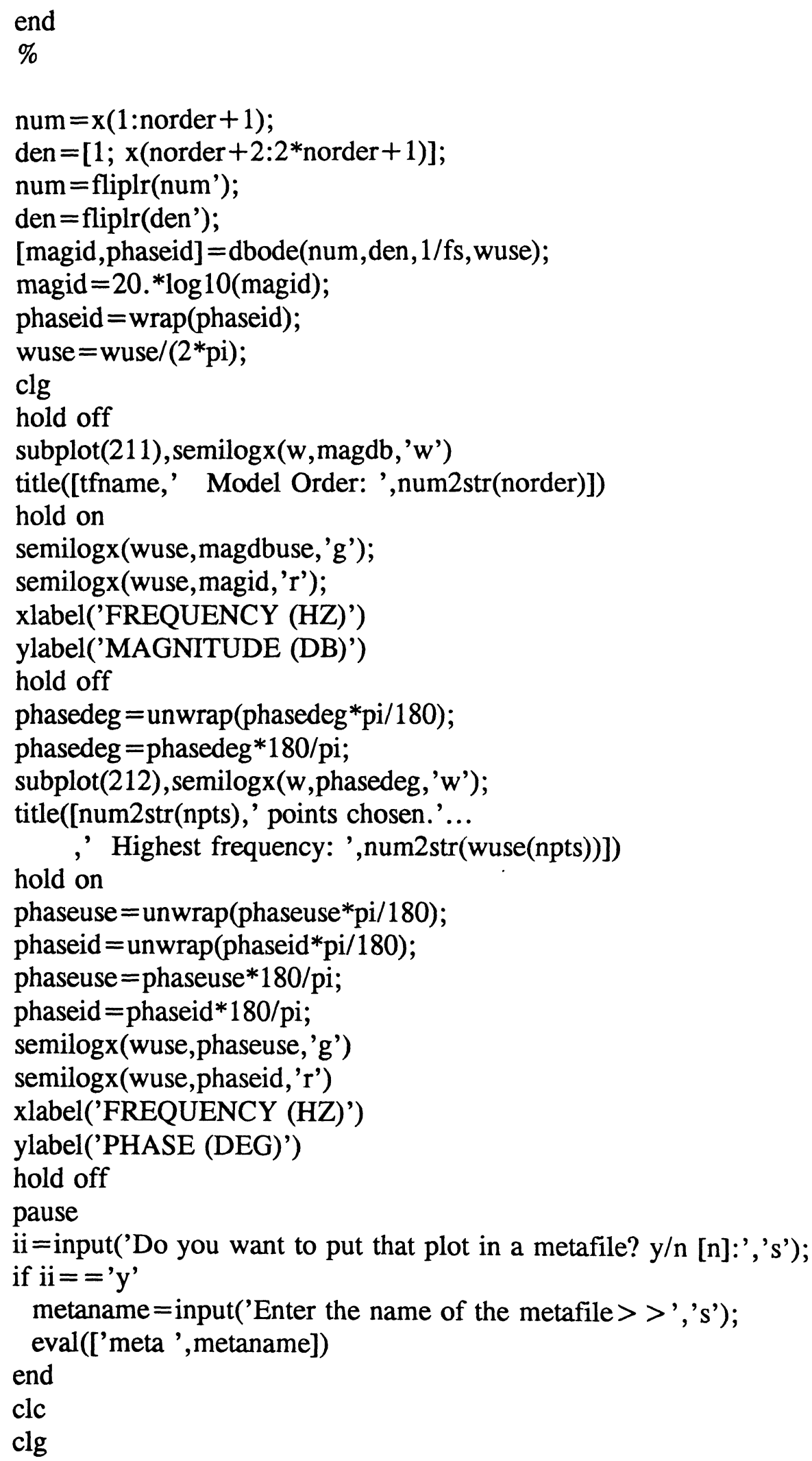




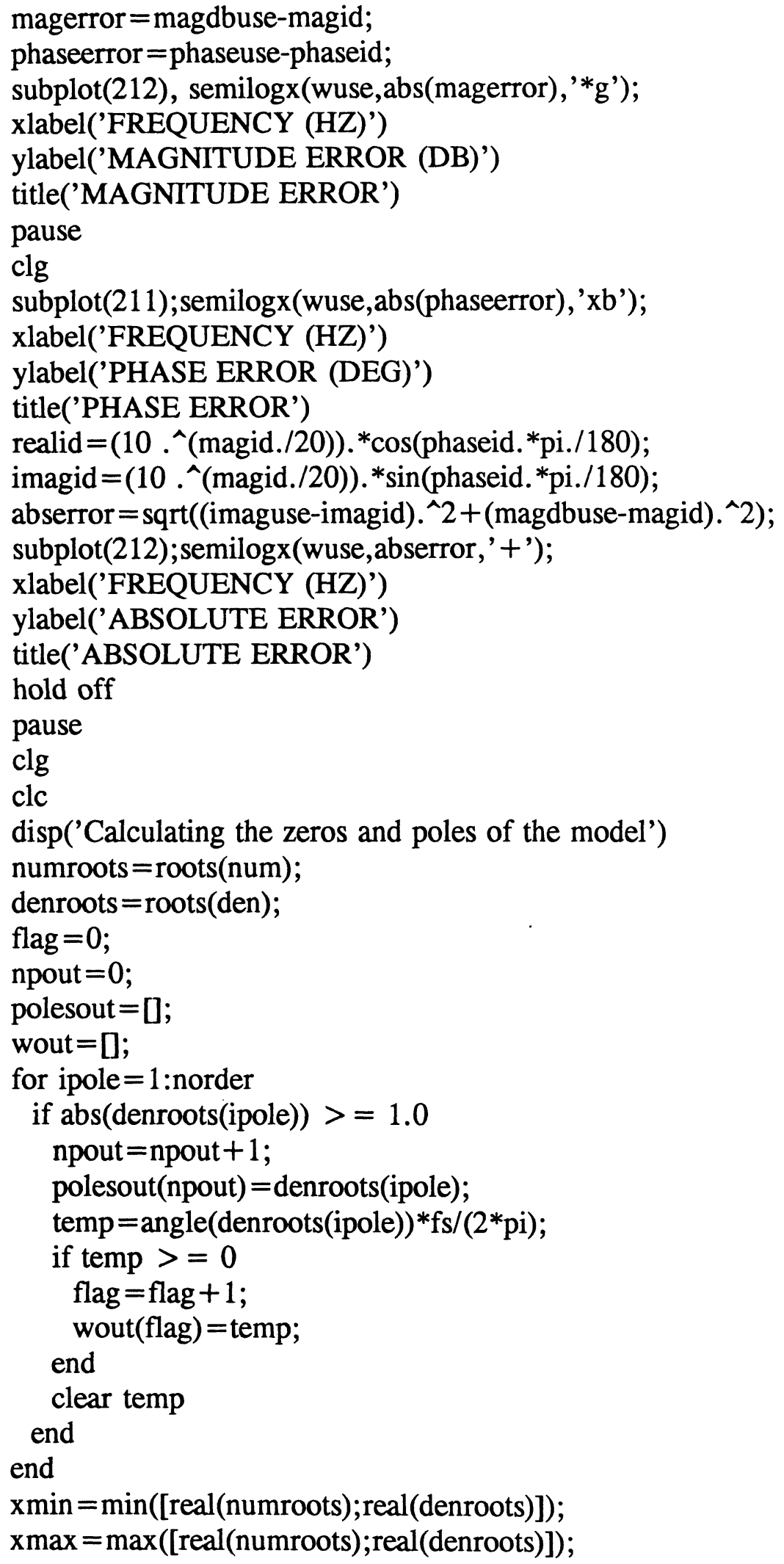




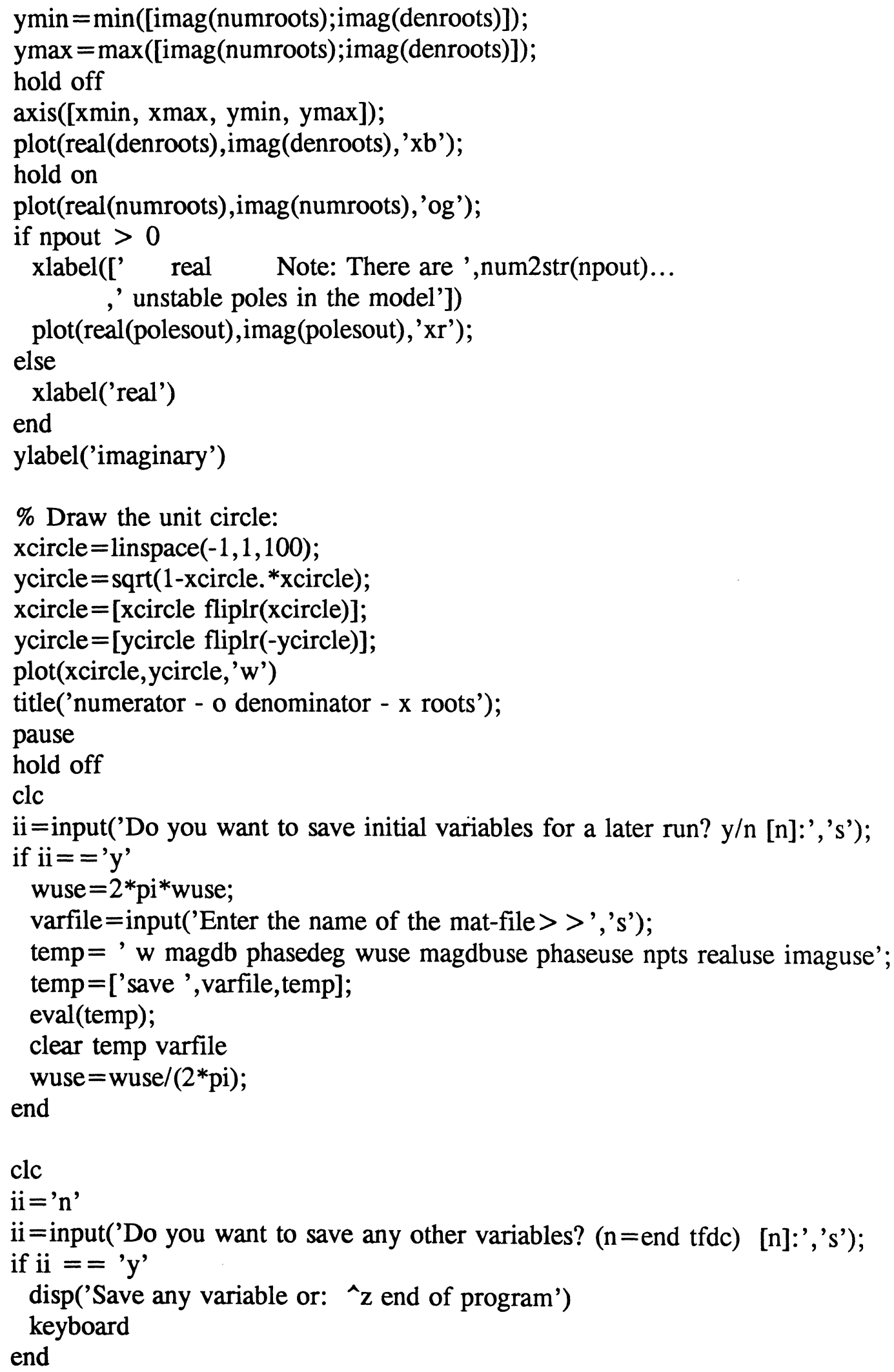

\% Draw the unit circle: 


\section{A.2. "TLSSOLVR.M"}

\% M-file to solve the system of linear equations $\mathrm{Ax}=\mathrm{b}$ using total

$\%$ least squares method of solution.

$\%$

\% Use -- $[\mathrm{x}]=\operatorname{tlssolvr}(\mathrm{A}, \mathrm{b})$

$\%$

$\%$

function $[\mathrm{x}]=\operatorname{tlssolvr}(\mathrm{a}, \mathrm{b})$

[junk,n] $=\operatorname{size}(\mathrm{a})$;

$[\mathrm{u}, \mathrm{s}, \mathrm{v}]=\operatorname{svd}([\mathrm{a}, \mathrm{b}], 0)$;

$\mathbf{x}=-1 / \mathrm{v}(\mathrm{n}+1, \mathrm{n}+1) * \mathrm{v}(1: \mathrm{n}, \mathrm{n}+1)$; 


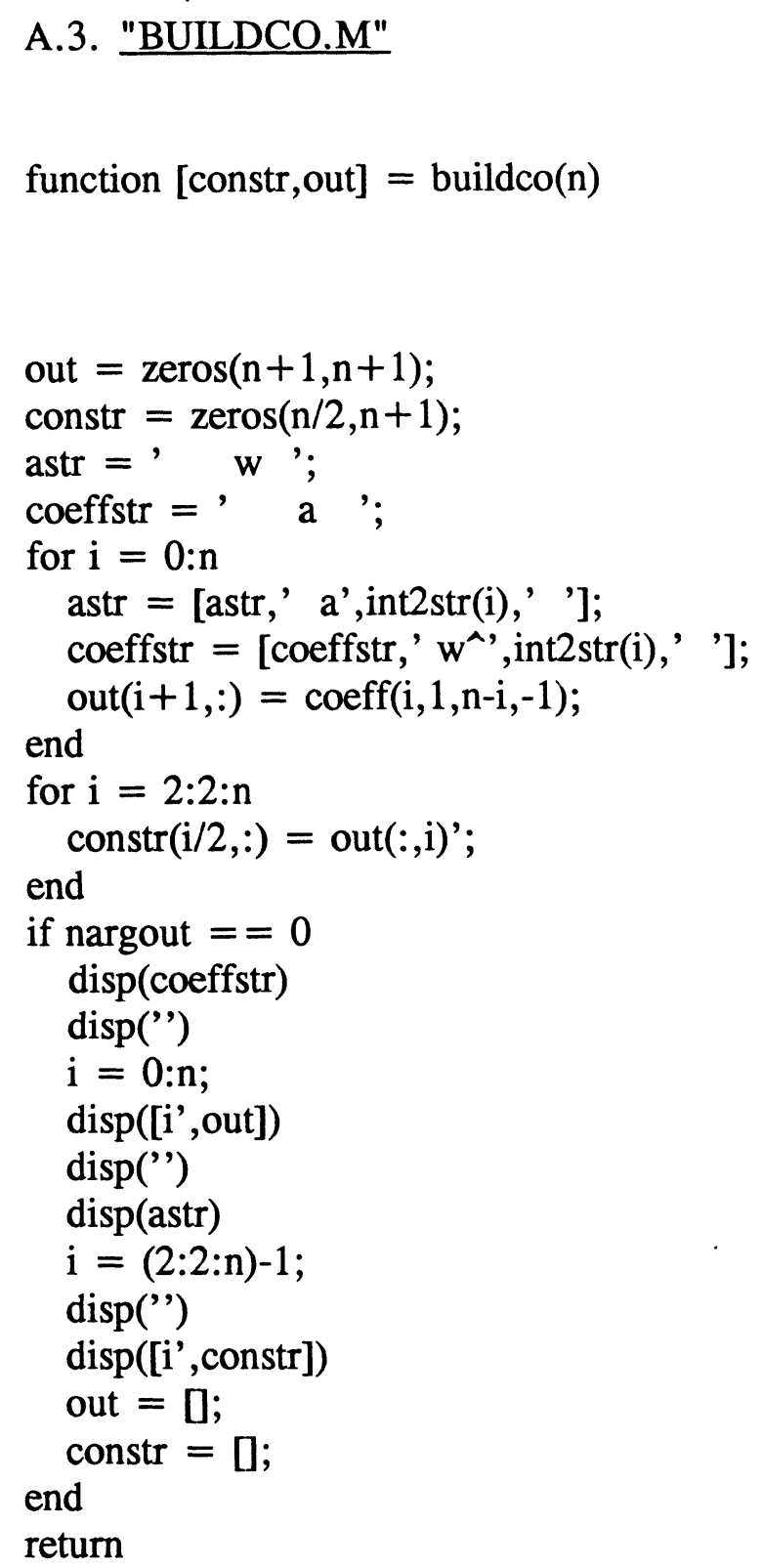




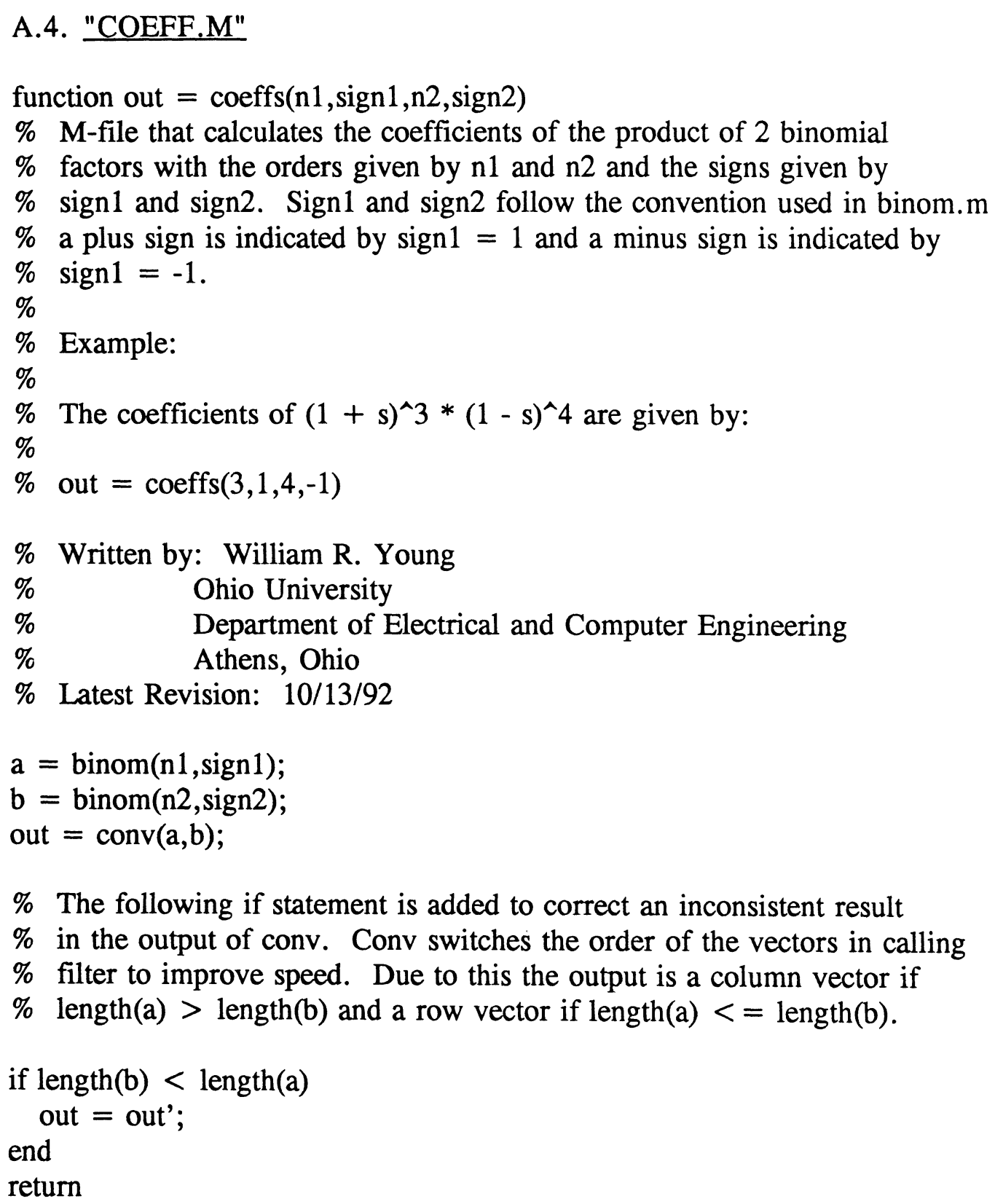




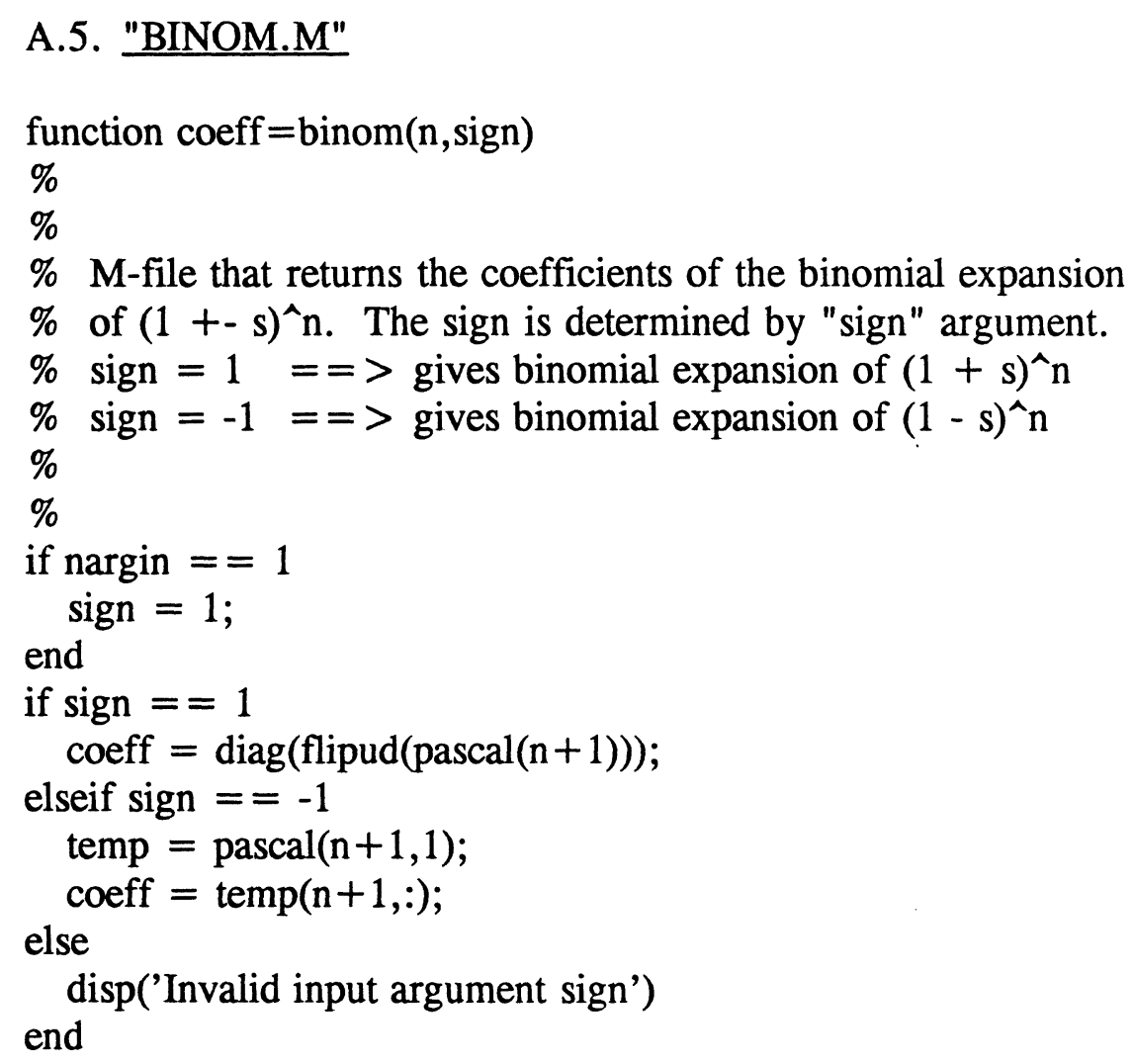

